KEK-TH-753

TUM-HEP-401/01

hep-ph/0103284

November 1, 2018

\title{
CP Violation in Tau Slepton Pair Production at Muon Colliders
}

\author{
S.Y. Choi ${ }^{1}$, M. Drees ${ }^{2}$, B. Gaissmaier ${ }^{2}$ and Jae Sik Lee ${ }^{3}$ \\ ${ }^{1}$ Department of Physics, Chonbuk National University, Chonju 561-756, Korea \\ ${ }^{2}$ Physik Dept., TU München, James Franck Str., D-85748 Garching, Germany \\ ${ }^{3}$ KEK Theory Group, Tsukuba, Ibaraki 305-0801, Japan
}

\begin{abstract}
We discuss in detail signals for $\mathrm{CP}$ violation in the Higgs boson and tau-slepton sectors through the production processes $\mu^{+} \mu^{-} \rightarrow \tilde{\tau}_{i}^{-} \tilde{\tau}_{j}^{+}$, where $i, j=1,2$ label the two $\tau$ slepton mass eigenstates in the minimal supersymmetric standard model. We assume that the soft breaking parameters of third generation sfermions contain $\mathrm{CP}$ violating phases, which induce $\mathrm{CP}$ violation in the Higgs sector through quantum corrections. We classify all the observables for probing $\mathrm{CP}$ violation in the Higgs boson and $\tau$ slepton sectors. These observables depend on the initial muon beam polarization, where we include transverse polarization states. If the heavy Higgs bosons can decay into tau slepton pairs, a complete determination of the CP properties of the neutral Higgs boson and $\tau$-slepton systems is possible. The interference between the Higgs boson and gauge boson contributions could also provide a powerful method for probing $\mathrm{CP}$ violation, if transversely polarized muon beams are available. We show in detail how to directly measure CP violation in the tau slepton system, under the assumption that the neutral Higgs mixing angles are determined through the on-shell production of the neutral Higgs bosons.
\end{abstract}

PACS number(s): 11.30.Pb, 11.30.Er 


\section{Introduction}

$\mathrm{CP}$ violation was observed in the neutral kaon system [1] and it is strongly suggested by recent experiments in $B$-meson decays [2]. In addition, $\mathrm{CP}$ violation constitutes one of the conditions for a dynamical generation of the cosmological baryon asymmetry [3]. In the Standard Model (SM), which contains only one physical neutral Higgs boson, the only source of CP violation is the complex phase of the quark mixing matrix [4. On the other hand, models with multiple Higgs doublets can have additional sources of CP violation, e.g. neutral and/or charged Higgs bosons of no definite CP quantum number.

Supersymmetry (SUSY) is now widely regarded to be the most plausible extension of the SM; among other things, it stabilizes the gauge hierarchy [5] and allows for the Grand Unification of all known gauge interactions [6]. Of course, supersymmetry must be (softly) broken to be phenomenologically viable. In general this introduces a large number of unknown parameters, many of which can be complex [7]. $\mathrm{CP}$-violating phases associated with sfermions of the first and, to a lesser extent, second generation are severely constrained by bounds on the electric dipole moments of the electron, neutron and muon. However, it has recently been realized [8] that cancellations between different diagrams allow some combinations of these phases to be quite large. Even in models with universal boundary conditions for soft breaking masses at some very high energy scale, the relative phase between the supersymmetric higgsino mass parameter $\mu$ and the universal trilinear soft breaking parameter $A_{0}$ can be $\mathcal{O}(1)$ [9]. If universality is not assumed, the phases of third generation trilinear soft breaking parameters are essentially unconstrained. In fact, some of these phases might be large [10 so as to provide non-SM sources of $\mathrm{CP}$ violation required for a dynamical generation of the baryon asymmetry of the Universe.

Recently it has been realized that the explicit CP violation in the mass matrices of the third generation squarks with such possibly large CP phasesf can induce sizable CP violation in the MSSM Higgs sector through loop corrections [12, 13, 14, 15, 16]. This induced CP violation in the MSSM Higgs sector can affect the phenomenology of the Higgs bosons at present and future colliders [12, 13, 16, 17, 18, 19, 20, 21]. These phases also directly affect the couplings of Higgs bosons to third generations sfermions. These couplings play an important role in the calculation of loop-induced couplings of Higgs bosons to photons and gluons as well as in the production of third generation sfermions at $\mu^{+} \mu^{-}$colliders [17].

In the past few years a considerable amount of effort has been devoted to investigations of the physics potential of high energy $\mu^{+} \mu^{-}$colliders [22] as a plausible future experimental program. Since muons emit far less synchrotron radiation than electrons do, a muon collider might be significantly smaller and cheaper than an $e^{+} e^{-}$collider operating at the same centerof-mass energy $\sqrt{s}$. The main physics advantage of muon colliders compared to the conventional $e^{+} e^{-}$and hadron colliders is that the larger Yukawa coupling of muons in many cases admits

\footnotetext{
${ }^{\dagger}$ In principle CP could also be violated in the SM by the QCD $\theta$-term, but bounds on the electric dipole moment of the neutron force $\theta_{\mathrm{QCD}}$ to be very small $\lesssim 10^{-10}$.

${ }^{\ddagger}$ If $\tan \beta$ is large, the third-generation $\mathrm{CP}$ phases are constrained by the electric dipole moments of the electron and neutron because in this case their contributions at the two-loop level could be sizable [11].
} 
copious production of Higgs bosons as $s$-channel resonances, allowing to perform precision measurements of their properties [22, 23, 24, 17, 25, 26]. In particular, they can allow us to search for $\mathrm{CP}$-violation in the couplings of Higgs bosons to not only heavy SM fermions but also to third generation sfermions, which have large Yukawa couplings.

It is difficult to probe these CP phases through processes controlled by gauge interactions, where large $\mathrm{CP}$-odd asymmetries can emerge only if some sfermion mass eigenstates are closely degenerate, with mass splitting of the order of the decay width, in which case flavor or chirality oscillations can occur [27, 28, 29. On the other hand, in the MSSM CP-violating phases can appear at tree level in the couplings of a single sfermion species to neutral Higgs bosons. These phases can give rise to large $\mathrm{CP}-$ odd asymmetries regardless of sfermion mass splittings. Here we focus on $\tilde{\tau}$ pair production. Unlike sfermions of the first two generations, $\tilde{\tau}$ 's generally have sizable couplings to heavy Higgs bosons even if the latter are much heavier than $m_{Z}$. Furthermore, unlike for $\tilde{b}$ and $\tilde{t}$ production the charge of a produced $\tilde{\tau}$ is usually readily measurable; this is necessary for the construction of most $\mathrm{CP}$-odd asymmetries. Finally, in most models sleptons are significantly lighter than squarks, making it easier to study them at lepton colliders.

In the present work we extend the previous work [17] significantly in order to discuss in more detail signals for CP violation in the production processes $\mu^{-} \mu^{+} \rightarrow \tilde{\tau}_{i}^{-} \tilde{\tau}_{j}^{+}$where $i, j=1,2$ label the two $\tau$ slepton mass eigenstates. We work in the framework of the MSSM with explicit CP violation. More specifically, we present a general formalism and a detailed analysis of the effects of the $\mathrm{CP}$-violating Higgs boson mixing, and of the $\mathrm{CP}$ phases in the tau slepton mass matrix, on the polarized cross section for tau-slepton pair production. We consider both longitudinal and transverse polarization of the initial muon and anti-muon beams. A detailed procedure is suggested for measuring CP violation directly in the tau slepton system, under the assumption that the neutral Higgs mixing angles are determined through the on-shell production of the neutral Higgs bosons as demonstrated in Ref. [21]. The polarization observables allow for the complete determination of the CP properties of the neutral Higgs boson and $\tau$ slepton systems. The interference between Higgs and gauge boson exchange contributions plays a crucial role in this analysis.

The remainder of this article is organized as follows. Section 2 is devoted to a brief review of the mass spectra and mixing patterns of the neutral Higgs bosons and tau sleptons, focusing on the role of the $\mathrm{CP}$ phases [13, 15, 16]. In Sec. 3 we present the helicity amplitudes of the production of a $\tau$-slepton pair in $\mu^{+} \mu^{-}$collisions with polarized muon beams. We also give a complete classification of the $\mathrm{CP}$ and $\mathrm{CP} \tilde{\mathrm{T}}$ transformation properties of the polarization observables. In Sec. 团we show how to extract the rate and polarization asymmetries by taking appropriate configurations of the muon and anti-muon spins. We then perform a detailed numerical analysis for a representative SUSY parameter set so as to get a concrete estimate of the relative usefulness of those observables. Section 6 is devoted to a brief summary of our findings and to conclusions. 


\section{Supersymmetric Particle Mixing}

Bounds on $\mathrm{CP}$ violating flavor changing processes tell us that the $\mathrm{CP}$ violating phases associated with flavor off-diagonal trilinear scalar interactions must be strongly suppressed [30]. We therefore neglect all these flavor changing $\mathrm{CP}$ violating phases in the present work, so that the scalar soft mass matrices and trilinear parameters are flavor diagonal and the complex trilinear terms are proportional to the corresponding fermion Yukawa couplings. Clearly the Yukawa interactions of the top and bottom (s)quarks play the most significant role in radiative corrections to the Higgs sector. Furthermore, for large values of $\tan \beta$ the $\tilde{\tau}_{L}$ and $\tilde{\tau}_{R}$ states are expected to mix strongly.

\section{1 $\quad \mathrm{CP}-$ violating neutral Higgs boson mixing}

In this section, we give a brief review of the calculation [15 of the Higgs boson mass matrix based on the full one-loop effective potential, valid for all values of the relevant third-generation squark soft-breaking parameters. The $\mathrm{CP}$-violating phases in the top and bottom squark sectors cause scalar-pseudoscalar mixing at one-loop level.

The MSSM contains two Higgs doublets $H_{1}, H_{2}$, with hypercharges $Y\left(H_{1}\right)=-Y\left(H_{2}\right)=$ $-1 / 2$. Here we are only interested in the neutral components, which we write as

$$
H_{1}^{0}=\frac{1}{\sqrt{2}}\left(\phi_{1}+i a_{1}\right) ; \quad H_{2}^{0}=\frac{\mathrm{e}^{i \xi}}{\sqrt{2}}\left(\phi_{2}+i a_{2}\right),
$$

where $\phi_{1,2}$ and $a_{1,2}$ are real fields. The constant phase $\xi$ can be set to zero at tree level, but will in general become non-zero once loop corrections are included.

The mass matrix of the neutral Higgs bosons can be computed from the effective potential 31

$$
\begin{aligned}
V_{\text {Higgs }} & =\frac{1}{2} m_{1}^{2}\left(\phi_{1}^{2}+a_{1}^{2}\right)+\frac{1}{2} m_{2}^{2}\left(\phi_{2}^{2}+a_{2}^{2}\right)-\left|m_{12}^{2}\right|\left(\phi_{1} \phi_{2}-a_{1} a_{2}\right) \cos \left(\xi+\theta_{12}\right) \\
+ & \left|m_{12}^{2}\right|\left(\phi_{1} a_{2}+\phi_{2} a_{1}\right) \sin \left(\xi+\theta_{12}\right)+\frac{\hat{g}^{2}}{8} \mathcal{D}^{2}+\frac{1}{64 \pi^{2}} \operatorname{Str}\left[\mathcal{M}^{4}\left(\log \frac{\mathcal{M}^{2}}{Q^{2}}-\frac{3}{2}\right)\right] .
\end{aligned}
$$

We have allowed the soft breaking parameter $m_{12}^{2}=\left|m_{12}^{2}\right| \mathrm{e}^{i \theta_{12}}$ to be complex, and we have introduced the quantities

$$
\mathcal{D}=\phi_{2}^{2}+a_{2}^{2}-\phi_{1}^{2}-a_{1}^{2} ; \quad \hat{g}^{2}=\frac{g^{2}+g^{\prime 2}}{4}
$$

where the symbols $g$ and $g^{\prime}$ stand for the $\mathrm{SU}(2)_{L}$ and $\mathrm{U}(1)_{Y}$ gauge couplings, respectively. $Q$ in Eq. (2) is the renormalization scale; the parameters of the tree-level potential, in particular the mass parameters $m_{1}^{2}, m_{2}^{2}$ and $m_{12}^{2}$, are running parameters, taken at scale $Q$. The potential (2) is then independent of $Q$, up to two-loop corrections.

The matrix $\mathcal{M}$ in Eq. (2) is the field-dependent mass matrix of all modes that couple to the Higgs bosons. The by far dominant contributions come from the third generation quarks 
and squarks. The (real) masses of the former are given by

$$
m_{b}^{2}=\frac{1}{2}\left|h_{b}\right|^{2}\left(\phi_{1}^{2}+a_{1}^{2}\right) ; \quad m_{t}^{2}=\frac{1}{2}\left|h_{t}\right|^{2}\left(\phi_{2}^{2}+a_{2}^{2}\right),
$$

where $h_{b}$ and $h_{t}$ are the bottom and top Yukawa couplings. The corresponding squark mass matrices can be written as

$$
\begin{gathered}
\mathcal{M}_{\tilde{t}}^{2}=\left(\begin{array}{cc}
m_{\widetilde{Q}}^{2}+m_{t}^{2}-\frac{1}{8}\left(g^{2}-\frac{g^{\prime 2}}{3}\right) \mathcal{D} & -h_{t}^{*}\left[A_{t}^{*}\left(H_{2}^{0}\right)^{*}+\mu H_{1}^{0}\right] \\
-h_{t}\left[A_{t} H_{2}^{0}+\mu^{*}\left(H_{1}^{0}\right)^{*}\right] & m_{\widetilde{U}}^{2}+m_{t}^{2}-\frac{g^{\prime 2}}{6} \mathcal{D}
\end{array}\right), \\
\mathcal{M}_{\tilde{b}}^{2}=\left(\begin{array}{cc}
m_{\widetilde{Q}}^{2}+m_{b}^{2}+\frac{1}{8}\left(g^{2}+\frac{g^{\prime 2}}{3}\right) \mathcal{D} & -h_{b}^{*}\left[A_{b}^{*}\left(H_{1}^{0}\right)^{*}+\mu H_{2}^{0}\right] \\
-h_{b}\left[A_{b} H_{1}^{0}+\mu^{*}\left(H_{2}^{0}\right)^{*}\right] & m_{\widetilde{D}}^{2}+m_{b}^{2}+\frac{g^{\prime 2}}{12} \mathcal{D}
\end{array}\right) .
\end{gathered}
$$

Here, $H_{1}^{0}$ and $H_{2}^{0}$ are given by Eq. (11) while $m_{t}^{2}$ and $m_{b}^{2}$ are as in Eq. (雨) and $\mathcal{D}$ has been defined in Eq. (3). In Eq. (5) $m_{\widetilde{Q}}^{2}, m_{\widetilde{U}}^{2}$ and $m_{\widetilde{D}}^{2}$ are real soft breaking parameters, $A_{b}$ and $A_{t}$ are complex soft breaking parameters, and $\mu$ is the complex supersymmetric Higgs(ino) mass parameter.

The mass matrix of the neutral Higgs bosons can now be computed from the matrix of second derivatives of the potential (2), where $m_{1}^{2}, m_{2}^{2}$ and $m_{12}^{2} \sin \left(\xi+\theta_{12}\right)$ are determined by the stationarity conditionsi $\partial V / \partial \phi_{1}=\partial V / \partial \phi_{2}=\partial V / \partial a_{1}=0$. The massless state $G^{0}=$ $a_{1} \cos \beta-a_{2} \sin \beta$ is the would-be Goldstone mode "eaten" by the longitudinal $Z$ boson. We are thus left with a squared mass matrix $\mathcal{M}_{H}^{2}$ for the three states $a=a_{1} \sin \beta+a_{2} \cos \beta, \phi_{1}$ and $\phi_{2}$. This matrix is real and symmetric, i.e. it has 6 independent entries. CP violation is caused by the appearance of nonvanishing $a \phi_{1}$ or $a \phi_{2}$ entries of this mass matrix. The sizes of these entries are controlled by the quantities

$$
\Delta_{\tilde{t}}=\frac{\Im \mathrm{m}\left(A_{t} \mu \mathrm{e}^{i \xi}\right)}{m_{\tilde{t}_{2}}^{2}-m_{\tilde{t}_{1}}^{2}}, \quad \Delta_{\tilde{b}}=\frac{\Im \mathrm{m}\left(A_{b} \mu \mathrm{e}^{i \xi}\right)}{m_{\tilde{b}_{2}}^{2}-m_{\tilde{b}_{1}}^{2}} .
$$

Explicit expressions for $\mathcal{M}_{H}^{2}$ can be found in Ref. [15].

Since $\mathcal{M}_{H}^{2}$ is real and symmetric, it can be diagonalized with a three-dimensional orthogonal rotation matrix $O$;

$$
\left(\begin{array}{c}
a \\
\phi_{1} \\
\phi_{2}
\end{array}\right)=O\left(\begin{array}{c}
H_{1} \\
H_{2} \\
H_{3}
\end{array}\right)
$$

Our convention for the three mass eigenvalues is $m_{H_{1}} \leq m_{H_{2}} \leq m_{H_{3}}$. The loop-corrected neutral Higgs boson sector is thus determined by fixing the values of the following parameters: $m_{A}$, which becomes the mass of the CP-odd Higgs boson if CP is conserved, and $\tan \beta$ fix the

${ }^{\dagger}$ The condition $\partial V / \partial a_{2}=0$ gives the same constraint as $\partial V / \partial a_{1}=0$ [15. 
tree-level Higgs potential; and $\mu, A_{t}, A_{b}$ and the soft-breaking third generation sfermion masses $m_{\tilde{Q}}, m_{\tilde{U}}$, and $m_{\tilde{D}}$, which fix the third generation squark mass matrices. After minimization of the potential the rephasing invariant sum $\theta_{12}+\xi$ of the radiatively induced phase $\xi$ and the phase $\theta_{12}$ of the soft breaking parameter $m_{12}^{2}$ is no longer an independent parameter. physically meaningful CP phases in the Higgs sector are thus the phases of the re-phasing invariant combinations $A_{t} \mu \mathrm{e}^{i \xi}$ and $A_{b} \mu \mathrm{e}^{i \xi}$ appearing in Eqs. (6). We refer to Ref. [15] for further details on neutral Higgs boson mixing in the presence of $\mathrm{CP}$-violation. This mixing changes all the couplings of the Higgs fields so that the effects of $\mathrm{CP}$ violation in the Higgs sector can be probed through various processes [18, 19, 20, 21].

\subsection{Tau slepton mixing}

The mass matrix squared $\mathcal{M}_{\tilde{\tau}}^{2}$ for the left-/right-handed tau sleptons is given by

$$
\mathcal{M}_{\tilde{\tau}}^{2}=\left(\begin{array}{cc}
X_{\tau} & Z_{\tau} \mathrm{e}^{-i \phi_{\tau}} \\
Z_{\tau} \mathrm{e}^{i \phi_{\tau}} & Y_{\tau}
\end{array}\right),
$$

where the matrix elements are defined as

$$
\begin{aligned}
X_{\tau} & =m_{\tilde{\tau}_{L}}^{2}+m_{\tau}^{2}+\frac{1}{2}\left(m_{Z}^{2}-2 m_{W}^{2}\right) \cos 2 \beta, \\
Y_{\tau} & =m_{\tilde{\tau}_{R}}^{2}+m_{\tau}^{2}+\left(m_{W}^{2}-m_{Z}^{2}\right) \cos 2 \beta, \\
Z_{\tau} & =m_{\tau}\left|A_{\tau}^{*}+\mu \mathrm{e}^{i \xi} \tan \beta\right|, \\
\phi_{\tau} & =\arg \left(-A_{\tau}-\mu^{*} \mathrm{e}^{-i \xi} \tan \beta\right),
\end{aligned}
$$

where $m_{\tilde{\tau}_{L, R}}^{2}$ are the left/right-handed soft-SUSY-breaking tau slepton masses squared, respectively.

The mass matrix squared $\mathcal{M}_{\tilde{\tau}}^{2}$ can be diagonalized by a unitary transformation $U_{\tau}$ :

$$
U_{\tau}^{\dagger} \mathcal{M}_{\tilde{\tau}}^{2} U_{\tau}=\operatorname{diag}\left(m_{\tilde{\tau}_{1}}^{2}, m_{\tilde{\tau}_{2}}^{2}\right)
$$

with the mass ordering $m_{\tilde{\tau}_{1}} \leq m_{\tilde{\tau}_{2}}$ as a convention. The diagonalization matrix can be parameterized as

$$
U_{\tau}=\left(\begin{array}{cc}
\cos \theta_{\tau} & -\sin \theta_{\tau} \mathrm{e}^{-i \phi_{\tau}} \\
\sin \theta_{\tau} \mathrm{e}^{i \phi_{\tau}} & \cos \theta_{\tau}
\end{array}\right),
$$

\footnotetext{
${ }^{\ddagger}$ As discussed in [15], $\xi$ and $\theta_{12}$ are not separately physical parameters. For example, one or the other can be set to zero in certain phase conventions for the fields. Similar remarks hold for the phases of $A_{t}, A_{b}$ and $\mu$. Altogther there are only three rephasing invariant (i.e. physical) phases, which we write as $\theta_{12}+\xi, \arg \left(A_{t} \mu \mathrm{e}^{i \xi}\right)$ and $\arg \left(A_{b} \mu \mathrm{e}^{i \xi}\right)$. The minimization of the potential fixes one of these combinations, leaving two independent physical phases as free input parameters.
} 
taking the ranges, $-\pi / 2 \leq \theta_{\tau} \leq 0$ and $0 \leq \phi_{\tau} \leq 2 \pi$. The tau slepton mass eigenvalues and mixing angles are then given by

$$
\begin{aligned}
& m_{\tilde{\tau}_{1,2}}^{2}=\bar{M}_{\tilde{\tau}}^{2} \mp \frac{\Delta_{\tau}}{2}, \\
& \sin 2 \theta_{\tau}=-2 \frac{Z_{\tau}}{\Delta_{\tau}}, \quad \cos 2 \theta_{\tau}=-\frac{X_{\tau}-Y_{\tau}}{\Delta_{\tau}} .
\end{aligned}
$$

The phenomenological parameters $\bar{M}_{\tilde{\tau}}^{2}$ and $\Delta_{\tau}$ are related to the tau slepton masses as

$$
\begin{aligned}
\bar{M}_{\tilde{\tau}}^{2} & \equiv \frac{m_{\tilde{\tau}_{2}}^{2}+m_{\tilde{\tau}_{1}}^{2}}{2}=\frac{X_{\tau}+Y_{\tau}}{2}, \\
\Delta_{\tau} & \equiv m_{\tilde{\tau}_{2}}^{2}-m_{\tilde{\tau}_{1}}^{2}=\sqrt{\left(X_{\tau}-Y_{\tau}\right)^{2}+4 Z_{\tau}^{2}} .
\end{aligned}
$$

Clearly tau slepton left-right mixing is enhanced for large $\tan \beta$ and large $|\mu|$.

\section{Tau Slepton Pair Production}

In this section, we present all the Feynman rules needed for the production process $\mu^{+} \mu^{-} \rightarrow$ $\tilde{\tau}_{i}^{-} \tilde{\tau}_{j}^{+}(i, j=1,2)$, derive the production amplitudes, and describe how to obtain the polarized cross sections with initial muon and anti-muon polarizations. We then classify all the polarization and rate asymmetries according to their CP and CP $\tilde{\mathrm{T}}$ transformation properties, and discuss the CP $\tilde{\mathrm{T}}$-even and -odd combinations of the neutral Higgs boson propagators, neglecting the $Z$ boson width.

\subsection{Feynman rules}

The couplings of the $\gamma$ and the neutral gauge boson $Z$ to fermions in the MSSM is described by the same Lagrangian as in the SM:

$$
\mathcal{L}_{V f f}=-e Q_{f} \bar{f} \gamma_{\mu} f A^{\mu}-\frac{g}{c_{W}} \bar{f} \gamma_{\mu}\left[\left(T_{f 3}-Q_{f} s_{W}^{2}\right) P_{-}-Q_{f} s_{W}^{2} P_{+}\right] f Z^{\mu}
$$

with $e=g s_{W}$ and the chirality projection operators $P_{ \pm}=\left(1 \pm \gamma_{5}\right) / 2 . Q_{f}$ is the electric charge of fermion $f$ in units of the proton charge. The couplings of the neutral Higgs bosons to leptons and quarks are described by the Lagrangian

$$
\begin{aligned}
\mathcal{L}_{H f f}= & -\frac{h_{l}}{\sqrt{2}} \sum_{k=1}^{3} \bar{\ell}\left[O_{2 k}-i s_{\beta} O_{1 k} \gamma_{5}\right] \ell H_{k}-\frac{h_{d}}{\sqrt{2}} \sum_{k=1}^{3} \bar{d}\left[O_{2 k}-i s_{\beta} O_{1 k} \gamma_{5}\right] d H_{k} \\
& -\frac{h_{u}}{\sqrt{2}} \sum_{k=1}^{3} \bar{u}\left[O_{3 k}-i c_{\beta} O_{1 k} \gamma_{5}\right] u H_{k} .
\end{aligned}
$$


Here $h_{l}, h_{d}$, and $h_{u}$ are the lepton and quark Yukawa couplings:

$$
h_{l}=\frac{g m_{l}}{\sqrt{2} m_{W} c_{\beta}}, \quad h_{d}=\frac{g m_{d}}{\sqrt{2} m_{W} c_{\beta}}, \quad h_{u}=\frac{g m_{u}}{\sqrt{2} m_{W} s_{\beta}},
$$

respectively, with $c_{\beta} \equiv \cos \beta$ and $s_{\beta} \equiv \sin \beta$. It is then clear that all the neutral Higgs bosons couple dominantly to the third generation fermions $t, b$ and $\tau$ and they couple to a muon about 200 times more strongly than to an electron - the primary reason for having a muon collider.

The couplings of the $\gamma$ and the neutral gauge boson $Z$ to tau sleptons in the MSSM are described in the mass eigenstate basis by the Lagrangian

$$
\mathcal{L}_{V \tilde{\tau}_{i} \tilde{\tau}_{j}}=i e A^{\mu} \delta_{i j}\left(\tilde{\tau}_{i}^{*} \overleftrightarrow{\partial_{\mu}} \tilde{\tau}_{j}\right)-i \frac{g}{c_{W}} Z^{\mu} Q_{i j}^{Z}\left(\tilde{\tau}_{i}^{*} \overleftrightarrow{\partial_{\mu}} \tilde{\tau}_{j}\right)
$$

where $Q_{i j}^{Z}$ are expressed in terms of the tau slepton mixing matrix $U_{\tau}$ as

$$
Q_{i j}^{Z}=s_{W}^{2} \delta_{i j}-\frac{1}{2}\left(U_{\tau}\right)_{1 i}^{*}\left(U_{\tau}\right)_{1 j}
$$

On the other hand, the Feynman rules for the Higgs boson couplings to tau slepton pairs, involving all the mixing and phases and including the phase rotations of the scalar tau fields, can be written as

$$
\mathcal{L}_{H \tilde{\tau} \tilde{\tau}}=-\frac{g m_{Z}}{c_{W}} O_{\alpha k}\left(U_{\tau}\right)_{\beta i}^{*}\left(U_{\tau}\right)_{\gamma j} V_{\alpha ; \beta \gamma} H_{k} \tilde{\tau}_{i}^{*} \tilde{\tau}_{j} \equiv-\frac{g m_{Z}}{c_{W}} V_{k ; i j} H_{k} \tilde{\tau}_{i}^{*} \tilde{\tau}_{j}
$$

where $\alpha$ labels the three neutral Higgs boson interaction eigenstates $\left\{a, \phi_{1}, \phi_{2}\right\}$, and $\{\beta, \gamma\}$ denote the chiralities $\{L, R\}$ of the $\tilde{\tau}$ interaction eigenstates. The chiral couplings $V_{\alpha ; \beta \gamma}$ for the scalar tau leptons can be obtained in a rather tedious but straightforward way as

$$
\begin{aligned}
& V_{a}=\frac{m_{\tau}}{2 m_{Z}^{2}}\left(\begin{array}{cc}
0 & i\left(A_{\tau}^{*} \tan \beta-\mu \mathrm{e}^{i \xi}\right) \\
-i\left(A_{\tau} \tan \beta-\mu^{*} \mathrm{e}^{-i \xi}\right) & 0
\end{array}\right), \\
& V_{\phi_{1}}=c_{\beta}\left(\begin{array}{cc}
s_{W}^{2}-\frac{1}{2}+\frac{m_{\tau}^{2}}{m_{Z}^{2} c_{\beta}^{2}} & -\frac{m_{\tau} A_{\tau}^{*}}{2 m_{Z}^{2} c_{\beta}^{2}} \\
-\frac{m_{\tau} A_{\tau}}{2 m_{Z}^{2} c_{\beta}^{2}} & -s_{W}^{2}+\frac{m_{\tau}^{2}}{m_{Z}^{2} c_{\beta}^{2}}
\end{array}\right), \\
& V_{\phi_{2}}=-s_{\beta}\left(\begin{array}{cc}
s_{W}^{2}-\frac{1}{2} & \frac{m_{\tau} \mu \mathrm{e}^{i \xi}}{2 m_{Z}^{2} c_{\beta} s_{\beta}} \\
\frac{m_{\tau} \mu^{*} \mathrm{e}^{-i \xi}}{2 m_{Z}^{2} c_{\beta} s_{\beta}} & -s_{W}^{2}
\end{array}\right) .
\end{aligned}
$$

These $2 \times 2$ matrices determine the dimensionless couplings $V_{k ; i j}$ through the relation:

$$
V_{k ; i j}=O_{\alpha k}\left(U_{\tau}\right)_{\beta i}^{*}\left(U_{\tau}\right)_{\gamma j} V_{\alpha ; \beta \gamma}
$$



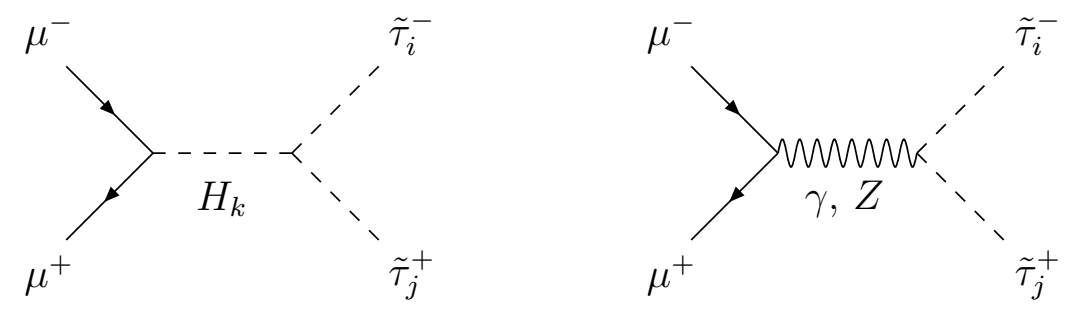

Figure 1: The mechanisms contributing to the process $\mu^{+} \mu^{-} \rightarrow \tilde{\tau}_{i}^{-} \tilde{\tau}_{j}^{+}:$three spin-0 neutralHiggs boson exchanges and spin-1 $\gamma$ and $Z$ exchanges. The indices $i, j$ are 1 or 2 , and the index $k$ is 1,2 or 3.

\subsection{Production amplitudes}

As shown in Fig. 1, the matrix element $\mathcal{M}_{i j}$ for $\mu^{+} \mu^{-} \rightarrow \tilde{\tau}_{i}^{-} \tilde{\tau}_{j}^{+}$receives contributions from $\gamma$ and $Z$ exchange as well as from the exchange of the neutral Higgs bosons of the MSSM. It can be written as:

$$
\mathcal{M}_{i j}=\frac{e^{2}}{s} \sum_{\alpha= \pm}\left\{Z_{\alpha i j}\left[\bar{v}(\bar{p})\left(\not p_{i}-\not p_{j}\right) P_{\alpha} u(p)\right]+m_{W} H_{\alpha i j}\left[\bar{v}(\bar{p}) P_{\alpha} u(p)\right]\right\}
$$

where $\alpha= \pm, P_{ \pm}=\left(1 \pm \gamma_{5}\right) / 2$, and the vector and scalar chiral couplings are given by

$$
\begin{aligned}
& Z_{-i j}=\delta_{i j}+D_{Z}(s) \frac{s_{W}^{2}-1 / 2}{c_{W}^{2} s_{W}^{2}} Q_{i j}^{Z}, \\
& Z_{+i j}=\delta_{i j}+D_{Z}(s) \frac{1}{c_{W}^{2}} Q_{i j}^{Z}, \\
& H_{-i j}=-Y_{\mu} \frac{D_{H_{k}}(s)}{c_{W}^{2} s_{W}^{2}} V_{k ; i j}\left[O_{2 k}+i s_{\beta} O_{1 k}\right], \\
& H_{+i j}=-Y_{\mu} \frac{D_{H_{k}}(s)}{c_{W}^{2} s_{W}^{2}} V_{k ; i j}\left[O_{2 k}-i s_{\beta} O_{1 k}\right] .
\end{aligned}
$$

The coupling $Y_{\mu}$, and the $Z$ and $H_{k}$ propagators $D_{Z}$ and $D_{H_{k}}(k=1,2,3)$ are:

$$
\begin{aligned}
Y_{\mu} & =\frac{m_{\mu}}{2 m_{W} c_{\beta}}, \\
D_{Z}(s) & =\frac{s}{s-m_{Z}^{2}+i m_{Z} \Gamma_{Z}}, \\
D_{H_{k}}(s) & =\frac{s}{s-m_{H_{k}}^{2}+i m_{H_{k}} \Gamma_{H_{k}}} .
\end{aligned}
$$


Defining the polar angle of the flight direction of the tau slepton $\tilde{\tau}_{i}^{-}$with respect to the $\mu^{-}$ beam direction by $\Theta$ (see Fig. 2), the explicit form of the production amplitude (22) can be evaluated in the helicity basis by the 2-component spinor technique of Ref. [32 [1. We denote the $\mu^{-}$and $\mu^{+}$helicities by $\sigma$ and $\bar{\sigma}$, with $\sigma=+$ and - standing for right- and left-handed particles, respectively. Neglecting the muon mass in the kinematics, the helicity amplitudes

$$
\mathcal{M}_{i j}(\sigma, \bar{\sigma}) \equiv e^{2}\langle\sigma \bar{\sigma}\rangle_{i j}
$$

read

$$
\begin{aligned}
\langle++\rangle_{i j} & =-\frac{m_{W}}{\sqrt{s}} H_{+i j}, & \langle+-\rangle_{i j} & =-Z_{+i j} \beta \sin \Theta, \\
\langle--\rangle_{i j} & =+\frac{m_{W}}{\sqrt{s}} H_{-i j}, & \langle-+\rangle_{i j} & =-Z_{-i j} \beta \sin \Theta .
\end{aligned}
$$

Here $\beta=\lambda^{1 / 2}\left(1, m_{\tilde{\tau}_{i}^{-}}^{2} / s, m_{\tilde{\tau}_{j}^{+}}^{2} / s\right)$, with $\lambda(x, y, z)=x^{2}+y^{2}+z^{2}-2(x y+y z+z x)$, describes the 3 -momentum of the scalar $\tau$ leptons in the center-of-mass frame. Note that in the limit of negligible kinematic mass of the muons vector boson exchange only contributes to configurations where $\mu^{-}$and $\mu^{+}$have opposite helicities, whereas Higgs boson exchange only contributes to configurations with equal $\mu^{+}$and $\mu^{-}$helicities. This implies that Higgs and gauge boson exchange contributions can only interfere with each other if at least one of the muons has nonvanishing transverse polarization, which can be understood as coherent superposition of left- and right-handed helicity states [32; see Eqs. (31) and (32) below.

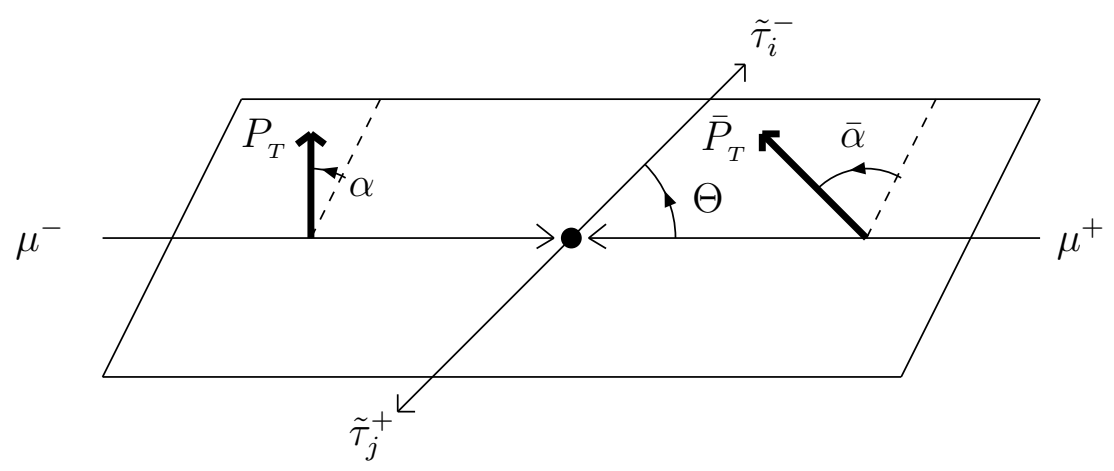

Figure 2: Schematic depiction of the production plane with the polar scattering angle $\Theta$. The transverse polarization vectors $P_{T}$ and $\bar{P}_{T}$ have azimuthal angles $\alpha$ and $\bar{\alpha}$ with respect to the event plane, respectively.

Before turning to the calculation of the cross section, let us briefly describe the symmetry properties of the helicity amplitudes. The CP transformation leads to a relation among the transition helicity amplitudes:

$$
\langle\sigma \bar{\sigma}\rangle_{i j} \stackrel{\mathrm{CP}}{\longleftrightarrow}(-1)(-1)^{(\sigma-\bar{\sigma}) / 2}\langle-\bar{\sigma},-\sigma\rangle_{j i}
$$

\footnotetext{
$\dagger$ Our convention for the momentum-dependent Weyl spinor for fermions going in $-z$ direction differs by an overall sign from that of Ref. 32]
} 
note that $\sigma-\bar{\sigma}$ gives the total spin, if the $\mu^{-}$momentum direction is used as quantization axis. Equivalently one has for the scalar and vector helicity amplitudes:

$$
\langle \pm \pm\rangle_{i j} \stackrel{\mathrm{CP}}{\longleftrightarrow}-\langle\mp \mp\rangle_{j i}, \quad\langle \pm \mp\rangle_{i j} \stackrel{\mathrm{CP}}{\longleftrightarrow}+\langle \pm \mp\rangle_{j i}
$$

Eqs. (28), (23) and (26) show that CP violation in diagonal channels $(i=j)$ can only occur in the presence of nonvanishing pseudoscalar couplings, since the contributions $\propto O_{1 k}$ in Eq. (23) are the only ones that violate relation (28). We will see in the next section that observable $\mathrm{CP}-$ odd effects can only arise in diagonal $\tilde{\tau}$ pair production if a pseudoscalar coupling interferes with one of the other couplings. On the other hand, all four sets of couplings (scalar, pseudoscalar, vector, and axial vector) can give rise to $\mathrm{CP}$ violation in non-diagonal $\tilde{\tau}$ pair production $(i \neq j)$.

Another useful classification is provided by the so-called "naive" time reversal $\tilde{T}$. Like the proper T-transformation, this transformation changes the directions of all 3-momenta and spin vectors, i.e. it leaves helicities unchanged. However, unlike a $\mathrm{T}$ transformation, a $\tilde{\mathrm{T}}$ transformation does not interchange initial and final states. In our case a $\tilde{\mathrm{T}}$ transformation simply corresponds to taking the complex conjugate of the helicity amplitudes. Under the combined CP $\tilde{\mathrm{T}}$ transformation the helicity amplitudes therefore transform as follows:

$$
\langle \pm \pm\rangle_{i j} \stackrel{\mathrm{CP} \tilde{\mathrm{T}}}{\longleftrightarrow}-\langle\mp \mp\rangle_{j i}^{*}, \quad\langle \pm \mp\rangle_{i j} \stackrel{\mathrm{CP} \tilde{\mathrm{T}}}{\longleftrightarrow}+\langle \pm \mp\rangle_{j i}^{*}
$$

We note that tree-level CP $\tilde{T}$ violation is only possible in the presence of finite $Z$ or Higgs boson widths. It is therefore very useful to analyze the CP and CPT properties of any physical observable simultaneously, so as to investigate not only $\mathrm{CP}$ violation itself but also its dependence on the real or imaginary part of some product(s) of propagators.

\subsection{Polarized production cross sections}

The production cross section for general (longitudinal or transverse) beam polarization can be computed either using standard trace techniques (employing general spin projection operators), or from the helicity amplitudes by a suitable rotation [32 from the helicity basis to a general spin basis. In the former case, neglecting the muon mass in the spin projection operators we can obtain the following approximated form for the $\mu^{\mp}$ projection operators:

$$
\begin{aligned}
& \frac{1}{2}(\not p+m)\left(1+\gamma_{5} \not s\right) \longrightarrow \frac{1}{2}\left(1+P_{L} \gamma_{5}\right) \not p+\frac{1}{2} \gamma_{5} P_{T}\left(\cos \alpha \not h_{1}+\sin \alpha \not h_{2}\right) \not p, \\
& \frac{1}{2}(\not p-m)\left(1+\gamma_{5} \not \supset\right) \longrightarrow \frac{1}{2}\left(1-\bar{P}_{L} \gamma_{5}\right) \not \triangleright+\frac{1}{2} \gamma_{5} \bar{P}_{T}\left(\cos \bar{\alpha} \not h_{1}+\sin \bar{\alpha} \not n_{2}\right) \not p .
\end{aligned}
$$

Here $s_{\mu}$ is the spin 4 -vector, and $n_{1 \mu}$ and $n_{2 \mu}$ are purely spatial vectors satisfying $n_{i} \cdot n_{j}=-\delta_{i j}$ and $n_{i} \cdot p=n_{i} \cdot \bar{p}=0 . P_{L}$ and $\bar{P}_{L}$ are the longitudinal polarizations of the $\mu^{-}$and $\mu^{+}$beams, while $P_{T}$ and $\bar{P}_{T}$ are the degrees of transverse polarization with $\alpha$ and $\bar{\alpha}$ being the azimuthal angles between the transverse polarization vectors and the momentum vector of $\tilde{\tau}_{i}^{-}$as shown in Fig. 2. We also note that $P_{L}^{2}+P_{T}^{2} \leq 1$ and $\bar{P}_{L}^{2}+\bar{P}_{T}^{2} \leq 1$. 

by

Equivalently the polarization weighted matrix element squared in the helicity basis is given

$$
\bar{\sum}|\mathcal{M}|^{2}=\sum_{\sigma \sigma^{\prime} \bar{\sigma} \bar{\sigma}^{\prime}} \mathcal{M}_{\sigma \bar{\sigma}} \mathcal{M}_{\sigma^{\prime} \bar{\sigma}^{\prime}}^{*} \rho_{\sigma \sigma^{\prime}}^{-} \rho_{\bar{\sigma} \bar{\sigma}^{\prime}}^{+}=\operatorname{Tr}\left[\mathcal{M} \rho^{+} \mathcal{M}^{\dagger} \rho^{-T}\right]
$$

where $\mathcal{M}_{\sigma \bar{\sigma}}(\sigma, \bar{\sigma}= \pm)$ denotes the helicity amplitude for any given production process $\mu^{-}(\sigma) \mu^{+}(\bar{\sigma}) \rightarrow$ $X$ and the $2 \times 2$ matrices $\rho^{\mp}$ are the polarization density matrices for the initial $\mu^{\mp}$ beams:

$$
\rho^{-}=\frac{1}{2}\left(\begin{array}{cc}
1+P_{L} & P_{T} \mathrm{e}^{-i \alpha} \\
P_{T} \mathrm{e}^{i \alpha} & 1-P_{L}
\end{array}\right), \quad \rho^{+}=\frac{1}{2}\left(\begin{array}{cc}
1+\bar{P}_{L} & -\bar{P}_{T} \mathrm{e}^{i \bar{\alpha}} \\
-\bar{P}_{T} \mathrm{e}^{-i \bar{\alpha}} & 1-\bar{P}_{L}
\end{array}\right)
$$

Applying the projection operators (30) or/and evaluating the trace (31) leads to the following matrix element squared for polarized $\mu^{+} \mu^{-} \rightarrow \tilde{\tau}_{i}^{-} \tilde{\tau}_{j}^{+}$production:

$$
\begin{aligned}
\Sigma_{i j} \equiv & \sum_{\sigma \sigma^{\prime} \bar{\sigma} \bar{\sigma}^{\prime}}\langle\sigma \bar{\sigma}\rangle_{i j}\left\langle\sigma^{\prime} \bar{\sigma}^{\prime}\right\rangle_{i j}^{*} \rho_{\sigma \sigma^{\prime}}^{-} \rho_{\bar{\sigma} \bar{\sigma}^{\prime}}^{+} \\
& =\left(1-P_{L} \bar{P}_{L}\right) C_{1}[i j]+\left(P_{L}-\bar{P}_{L}\right) C_{2}[i j] \\
& +\left(1+P_{L} \bar{P}_{L}\right) C_{3}[i j]+\left(P_{L}+\bar{P}_{L}\right) C_{4}[i j] \\
& +\left(P_{T} \cos \alpha+\bar{P}_{T} \cos \bar{\alpha}\right) C_{5}[i j]+\left(P_{T} \sin \alpha+\bar{P}_{T} \sin \bar{\alpha}\right) C_{6}[i j] \\
& +\left(P_{T} \cos \alpha-\bar{P}_{T} \cos \bar{\alpha}\right) C_{7}[i j]+\left(P_{T} \sin \alpha-\bar{P}_{T} \sin \bar{\alpha}\right) C_{8}[i j] \\
& +\left(P_{L} \bar{P}_{T} \cos \bar{\alpha}+\bar{P}_{L} P_{T} \cos \alpha\right) C_{9}[i j]+\left(P_{L} \bar{P}_{T} \sin \bar{\alpha}+\bar{P}_{L} P_{T} \sin \alpha\right) C_{10}[i j] \\
& +\left(P_{L} \bar{P}_{T} \cos \bar{\alpha}-\bar{P}_{L} P_{T} \cos \alpha\right) C_{11}[i j]+\left(P_{L} \bar{P}_{T} \sin \bar{\alpha}-\bar{P}_{L} P_{T} \sin \alpha\right) C_{12}[i j] \\
& +P_{T} \bar{P}_{T}\left[\cos (\alpha+\bar{\alpha}) C_{13}[i j]+\sin (\alpha+\bar{\alpha}) C_{14}[i j]\right] \\
& +P_{T} \bar{P}_{T}\left[\cos (\alpha-\bar{\alpha}) C_{15}[i j]+\sin (\alpha-\bar{\alpha}) C_{16}[i j]\right] .
\end{aligned}
$$

The coefficients $C_{n}(n=1-16)$ are defined in terms of the helicity amplitudes by

$$
\begin{aligned}
& C_{1}=\frac{1}{4}\left[|\langle+-\rangle|^{2}+|\langle-+\rangle|^{2}\right], \quad C_{2}=\frac{1}{4}\left[|\langle+-\rangle|^{2}-|\langle-+\rangle|^{2}\right], \\
& C_{3}=\frac{1}{4}\left[|\langle++\rangle|^{2}+|\langle--\rangle|^{2}\right], \quad C_{4}=\frac{1}{4}\left[|\langle++\rangle|^{2}-|\langle--\rangle|^{2}\right], \\
& C_{5}=\frac{1}{4} \Re \mathrm{e}(\langle++\rangle-\langle--\rangle)(\langle-+\rangle-\langle+-\rangle)^{*}, \\
& C_{6}=\frac{1}{4} \Im \mathrm{m}(\langle++\rangle-\langle--\rangle)(\langle-+\rangle+\langle+-\rangle)^{*}, \\
& C_{7}=\frac{1}{4} \Re \mathrm{e}(\langle++\rangle+\langle--\rangle)(\langle-+\rangle+\langle+-\rangle)^{*}, \\
& C_{8}=\frac{1}{4} \Im \mathrm{m}(\langle++\rangle+\langle--\rangle)(\langle-+\rangle-\langle+-\rangle)^{*}, \\
& C_{9}=\frac{1}{4} \Re \mathrm{e}(\langle++\rangle+\langle--\rangle)(\langle-+\rangle-\langle+-\rangle)^{*},
\end{aligned}
$$




$$
\begin{aligned}
C_{10} & =\frac{1}{4} \Im \mathrm{m}(\langle--\rangle+\langle++\rangle)(\langle-+\rangle+\langle+-\rangle)^{*}, \\
C_{11} & =\frac{1}{4} \Re \mathrm{e}(\langle--\rangle-\langle++\rangle)(\langle-+\rangle+\langle+-\rangle)^{*}, \\
C_{12} & =\frac{1}{4} \Im \mathrm{m}(\langle++\rangle-\langle--\rangle)(\langle+-\rangle-\langle-+\rangle)^{*}, \\
C_{13} & =-\frac{1}{2} \Re \mathrm{e}\left[\langle-+\rangle\langle+-\rangle^{*}\right], \quad C_{14}=\frac{1}{2} \Im \mathrm{m}\left[\langle-+\rangle\langle+-\rangle^{*}\right], \\
C_{15} & =-\frac{1}{2} \Re \mathrm{e}\left[\langle--\rangle\langle++\rangle^{*}\right], \quad C_{16}=\frac{1}{2} \Im \mathrm{m}\left[\langle--\rangle\langle++\rangle^{*}\right] .
\end{aligned}
$$

The production cross section is then given in terms of the distribution $\Sigma_{i j}$ in Eq. (33) by

$$
\frac{d \sigma}{d \cos \Theta d \Phi}\left(\mu^{+} \mu^{-} \rightarrow \tilde{\tau}_{i}^{-} \tilde{\tau}_{j}^{+}\right)=\frac{\alpha^{2}}{4 s} \beta \Sigma_{i j}
$$

with $\beta$ as in Eqs. (26). The dependence of the distribution $\Sigma_{i j}$ on the azimuthal angle $\Phi$ of the production plane is encoded in the angles $\alpha$ and $\bar{\alpha}$. If the azimuthal angle $\Phi$ is measured with respect to the direction of the $\mu^{-}$transverse polarization vector, the $\Phi$ dependence can be exhibited explicitly by taking for the angles $\alpha$ and $\bar{\alpha}$

$$
\alpha=-\Phi, \quad \bar{\alpha}=\eta-\Phi,
$$

where $\eta$ is the rotational invariant difference $\bar{\alpha}-\alpha$ of the azimuthal angles of the $\mu^{+}$and $\mu^{-}$ transverse polarization vectors. The polarization coefficients can be classified according to their correlation patterns of the vector and scalar contributions as follows:

$$
\begin{aligned}
& \text { Vector and vector (VV) correlations : } C_{1}, C_{2}, C_{13}, C_{14}, \\
& \text { Scalar and scalar }(\mathrm{SS}) \text { correlations }: C_{3}, C_{4}, C_{15}, C_{16}, \\
& \text { Scalar and vector }(\mathrm{SV}) \text { correlations }: C_{5}, C_{6}, C_{7}, C_{8}, C_{9}, C_{10}, C_{11}, C_{12} .
\end{aligned}
$$

Among the sixteen observables, only six observables - the $2 \mathrm{VV}$ observables $\left\{C_{1}, C_{2}\right\}$ and the 4 SS observables $\left\{C_{3}, C_{4}, C_{15}, C_{16}\right\}$ - can be measured independently of the azimuthal angle $\Phi$, but the other 10 observables require the reconstruction of the production plane. This is true in particular for the observables involving scalar-vector correlations; as already stated in Sec. 3.2, these observables are only nonzero if at least one of the incoming beams has nonvanishing transverse polarization.

\subsection{Observables with definite $\mathrm{CP}$ and $\mathrm{CP} \tilde{\mathrm{T}}$ properties}

The CP and CP $\tilde{\mathrm{T}}$ transformations act on the polarization vectors of the initial muon beams according to the simultaneous exchanges:

$$
\begin{array}{llllllll}
P_{L} & \stackrel{\mathrm{CP}}{\longleftrightarrow}-\bar{P}_{L}, & P_{T} \quad \stackrel{\mathrm{CP}}{\longleftrightarrow} \bar{P}_{T} ; & \alpha & \stackrel{\mathrm{CP}}{\longleftrightarrow} & \bar{\alpha}, \\
P_{L} & \stackrel{\mathrm{CP} \tilde{\mathrm{T}}}{\longleftrightarrow}-\bar{P}_{L}, & P_{T} & \stackrel{\mathrm{CP} \tilde{\mathrm{T}}}{\longleftrightarrow} & \bar{P}_{T} ; & \alpha & \stackrel{\mathrm{CP} \tilde{\mathrm{T}}}{\longleftrightarrow}-\bar{\alpha} .
\end{array}
$$


The CP and CP $\tilde{\mathrm{T}}$ relations (38) of the polarization vectors lead to the following classification of the terms in Eq. (33): $7 \mathrm{CP}$-even and $\mathrm{CP} \tilde{\mathrm{T}}$-even, $3 \mathrm{CP}$-even and $\mathrm{CP} \tilde{\mathrm{T}}$-odd, $3 \mathrm{CP}$-odd and $\mathrm{CP} \tilde{\mathrm{T}}$-even, and $3 \mathrm{CP}$-odd and CP $\tilde{\mathrm{T}}$-odd polarization factors, as shown in Table 1 .

Table 1: The classification of the correlations of the muon and anti-muon polarization vectors that appear in Eq. (33) according to their CP and CP $\tilde{T}$ properties, based on the CP and CP $\tilde{T}$ relations (38).

\begin{tabular}{|c|c|c|}
\hline CP & CPT & Polarization factors \\
\hline \hline \multirow{2}{*}{ even } & even & $\begin{array}{l}1-P_{L} \bar{P}_{L}, \quad P_{L}-\bar{P}_{L}, \quad 1+P_{L} \bar{P}_{L}, \quad P_{T} \cos \alpha+\bar{P}_{T} \cos \bar{\alpha} \\
P_{L} \bar{P}_{T} \cos \bar{\alpha}-\bar{P}_{L} P_{T} \cos \alpha, P_{T} \bar{P}_{T} \cos (\alpha+\bar{\alpha}), P_{T} \bar{P}_{T} \cos (\alpha-\bar{\alpha})\end{array}$ \\
\cline { 2 - 4 } & odd & $P_{T} \sin \alpha+\bar{P}_{T} \sin \bar{\alpha}, P_{L} \bar{P}_{T} \sin \bar{\alpha}-\bar{P}_{L} P_{T} \sin \alpha, P_{T} \bar{P}_{T} \sin (\alpha+\bar{\alpha})$ \\
\hline \hline \multirow{2}{*}{ odd } & even & $P_{T} \sin \alpha-\bar{P}_{T} \sin \bar{\alpha}, P_{L} \bar{P}_{T} \sin \bar{\alpha}+\bar{P}_{L} P_{T} \sin \alpha, P_{T} \bar{P}_{T} \sin (\alpha-\bar{\alpha})$ \\
\cline { 2 - 4 } & odd & $P_{L}+\bar{P}_{L}, P_{T} \cos \alpha-\bar{P}_{T} \cos \bar{\alpha}, P_{L} \bar{P}_{T} \cos \bar{\alpha}+\bar{P}_{L} P_{T} \cos \alpha$ \\
\hline
\end{tabular}

The coefficients $C_{n}[i j](n=1-16)$ corresponding to these polarization factors are bilinears in $Z_{ \pm i j}$ and $H_{ \pm i j}$. For later convenience we define the asymmetric combinations [ $\left.C_{n}[12]\right]$ and the symmetric combinations $\left\{C_{n}[i j]\right\}(i j=11,12,22)$ as

$$
\left[C_{n}[12]\right] \equiv \frac{1}{2}\left(C_{n}[12]-C_{n}[21]\right), \quad\left\{C_{n}[i j]\right\} \equiv \frac{1}{2}\left(C_{n}[i j]+C_{n}[j i]\right) .
$$

Clearly the coefficients multiplying CP-even polarization factors (the first group in Table 1) can give rise to $\mathrm{CP}$ violation only through the antisymmetric combinations $\left[C_{n}[12]\right]$, which describe rate asymmetries. In contrast, all symmetric combinations $\left\{C_{n}[i j]\right\}$ of the coefficients of the second group in Table 1, which have $\mathrm{CP}$-odd polarization factors, can contribute to $\mathrm{CP}$-odd polarization or azimuthal angle asymmetries; these can be probed for three different $\mathrm{CP}$-even final states $\left(\tilde{\tau}_{i}^{-} \tilde{\tau}_{i}^{+}, i=1,2\right.$ and the sum of $\tilde{\tau}_{1}^{-} \tilde{\tau}_{2}^{+}$and $\tilde{\tau}_{1}^{+} \tilde{\tau}_{2}^{-}$production). Based on these observations one can classify all the 64 distributions according to their CP and CP $\tilde{T}$ parities as shown in Table 2. We will show in Sec. 4.2 that all 64 coefficients appearing in Eq. (33) can be extracted independently, if the polarization of both beams can be be controlled completely and the final tau slepton pair is identified.

We thus see that one can define $10 \mathrm{CP}$-violating rate asymmetries involving anti-symmetric combinations in the $\tilde{\tau}$ indices and 6 polarization/angle asymmetries involving symmetric combinations in the $\tilde{\tau}$ indices; we recall that the latter can be studied for three different final states, leading to a total of 28 different asymmetries. More detailed explanations of the definition of the rate and polarization asymmetries and of the extraction of the asymmetries by adjusting the muon and anti-muon polarizations will be given in the next section. 
Table 2: The classification of the coefficients $C_{n}[i j](n=1-16, i j=11,12,21,22)$ according to their $C P$ and $C P \tilde{T}$ properties. The underlined observables can be constructed without direct reconstruction of the scattering plane. Here, $\left\{C_{n}[i j]\right\}=\left(C_{n}[i j]+C_{n}[j i]\right) / 2$ and $\left[C_{n}[12]\right]=$ $\left(C_{n}[12]-C_{n}[21]\right) / 2$.

\begin{tabular}{|c|c|c|}
\hline CP & CPT & Coefficients $(i j=11,12,22)$ \\
\hline \hline \multirow{2}{*}{ even } & even & $\frac{\left\{C_{1}[i j]\right\}}{\left\{C_{15}[i j]\right\}}, \underline{\left\{C_{2}[i j]\right\}}, \underline{\left[C_{4}[12]\right]},\left[C_{3}[i j]\right\},\left\{C_{7}[12]\right],\left[C_{5}[i j]\right\},\left\{C_{11}[i j]\right\},\left\{C_{13}[i j]\right\}$ \\
\cline { 2 - 4 } & odd & $\left\{C_{6}[i j]\right\},\left\{C_{12}[i j]\right\},\left\{C_{14}[i j]\right\},\left[C_{8}[12]\right],\left[C_{10}[12]\right], \underline{\left[C_{16}[12]\right]}$ \\
\hline \hline \multirow{2}{*}{ odd } & even & $\left\{C_{8}[i j]\right\},\left\{C_{10}[i j]\right\}, \underline{\left\{C_{16}[i j]\right\}},\left[C_{6}[12]\right],\left[C_{12}[12]\right],\left[C_{14}[12]\right]$ \\
\cline { 2 - 3 } & odd & $\underline{\left\{C_{4}[i j]\right\}},\left\{C_{7}[i j]\right\},\left\{C_{9}[i j]\right\}, \underline{\left[C_{1}[12]\right],\left[C_{2}[12]\right],}, \underline{\left[C_{3}[12]\right]}$ \\
\hline$\left.C_{5}[12]\right],\left[C_{11}[12]\right],\left[C_{13}[12]\right], \underline{\left[C_{15}[12]\right]}$ \\
\hline
\end{tabular}

Any $\mathrm{CP} \tilde{\mathrm{T}}$-odd observable requires some $\mathrm{CP}$-preserving phase. At tree-level this can only be provided by the $Z$ and Higgs boson widths in the tau slepton pair production processes. Therefore, if the $Z$ width is neglected, in which case $Z_{ \pm i j}$ is Hermitian, the following six quantities involving only the $\gamma$ or $Z$ boson couplings to muons and tau sleptons vanish:

$$
\left[C_{1}[12]\right]=\left[C_{2}[12]\right]=\left[C_{13}[12]\right]=C_{14}[11]=C_{14}[22]=0 .
$$

Furthermore, Eqs. (18), (23) and (34) show that

$$
\left[C_{14}[12]\right]=\left\{C_{14}[12]\right\}=0,
$$

even for nonvanishing $\Gamma_{Z}$. All other $\mathrm{CP} \tilde{\mathrm{T}}$-odd quantities can get contributions from some Higgs boson width, as will be clarified in the next section.

\subsection{CP $\tilde{\mathrm{T}}-$-even and odd combinations of Higgs boson propagators}

The behavior of each observable under a CP $\tilde{T}$ transformation plays a crucial role in determining the interference pattern of the Higgs boson contributions, among themselves and with the $\gamma$ and $Z$ boson contributions to this observable. Every CP $\tilde{T}$-even (odd) observable involving only scalar contributions depends on the real (imaginary) parts of the products $D_{H_{k}} D_{H_{l}}^{*}$ of Higgs boson propagators; note that only $k \neq l$ gives a nonzero imaginary part. We already showed that the observable $C_{14}$ involving only the vector contributions is negligible, see Eqs. (40) and (41). In the approximation $\Gamma_{Z}=0$ all the vector boson contributions $Z_{-}$and $Z_{+}$in Eq. (23) are 
hermitian. As a result, the interference terms between scalar and vector contributions depend on the real or imaginary parts of the Higgs boson propagators $D_{H_{k}}$ themselves.

This discussion shows that it is convenient to introduce abbreviated notations for the real and imaginary parts:

$$
\begin{aligned}
& \mathcal{S}_{k l} \equiv \Re \mathrm{e}\left[D_{H_{k}} D_{H_{l}}^{*}\right]=s^{2} \frac{\left(s-m_{H_{k}}^{2}\right)\left(s-m_{H_{l}}^{2}\right)+m_{H_{k}} m_{H_{l}} \Gamma_{H_{k}} \Gamma_{H_{l}}}{\left[\left(s-m_{H_{k}}^{2}\right)^{2}+m_{H_{k}}^{2} \Gamma_{H_{k}}^{2}\right]\left[\left(s-m_{H_{l}}^{2}\right)^{2}+m_{H_{l}}^{2} \Gamma_{H_{l}}^{2}\right]}, \\
& \mathcal{D}_{k l} \equiv \Im \mathrm{m}\left[D_{H_{k}} D_{H_{l}}^{*}\right]=s^{2} \frac{\left(s-m_{H_{k}}^{2}\right) m_{H_{l}} \Gamma_{H_{l}}-\left(s-m_{H_{l}}^{2}\right) m_{H_{k}} \Gamma_{H_{k}}}{\left[\left(s-m_{H_{k}}^{2}\right)^{2}+m_{H_{k}}^{2} \Gamma_{H_{k}}^{2}\right]\left[\left(s-m_{H_{l}}^{2}\right)^{2}+m_{H_{l}}^{2} \Gamma_{H_{l}}^{2}\right]}
\end{aligned}
$$

It is worthwhile to note two points: (a) the denominator reveals a typical two-pole structure so that the Higgs boson contributions are greatly enhanced near the poles; (b) the numerator of $\mathcal{S}_{k l}$ is negative between two resonances with a mass splitting larger than their typical widths but positive otherwise. In contrast, for realistic widths of the MSSM Higgs bosons the numerator of $\mathcal{D}_{k l}$ is always positive (negative) if $m_{H_{l}} \geq m_{H_{k}}\left(m_{H_{k}} \geq m_{H_{l}}\right)$. Moreover, it increases (decreases) linearly with $s$ if $m_{H_{l}} \Gamma_{H_{l}}>(<) m_{H_{k}} \Gamma_{H_{k}}$.

As noted earlier, in the approximation $\Gamma_{Z}=0$ the SV interference terms depend on the real and imaginary parts of each Higgs boson propagators $D_{H_{k}}$, which are given by

$$
\Re \mathrm{e}\left[D_{H_{k}}\right]=\frac{s\left(s-m_{H_{K}}^{2}\right)}{\left(s-m_{H_{k}}^{2}\right)^{2}+m_{H_{k}}^{2} \Gamma_{H_{k}}^{2}}, \quad \Im \mathrm{m}\left[D_{H_{k}}\right]=-\frac{s m_{H_{K}} \Gamma_{H_{k}}}{\left(s-m_{H_{k}}^{2}\right)^{2}+m_{H_{k}}^{2} \Gamma_{H_{k}}^{2}},
$$

respectively. Note that the real parts change their sign whenever the c.m. energy crosses the corresponding pole, but the imaginary parts are always negative.

Combining the coefficients from the mixing matrix elements with the corresponding propagatordependent factors enables us to straightforwardly understand the qualitative $\sqrt{s}$ dependence of the combinations of coefficients $C_{n}[i j]$ listed in Table 2. However, the energy dependence of the corresponding properly normalized asymmetries is also affected by the energy dependence of the normalization factors, which can be nontrivial. We will discuss these issues further in Sec. 5, where we present numerical results.

\section{Polarization and rate asymmetries}

In this section, we present the explicit forms of all the VV, SS, and SV polarization and rate correlations and then explain how to extract the corresponding asymmetries by adjusting initial beam polarization and, in some cases, inserting weight functions of the polar angle in the phase space integral. For completeness we include the CP-even correlations listed in the upper half of Table 2.

\subsection{Expressions for the correlations}




\subsubsection{Vector-vector correlations}

The VV correlations are very useful for reconstructing the tau slepton system independent of the Higgs boson system. In principle there exist 9 non-trivial $\mathrm{CP}$-even and $\mathrm{CP} \tilde{\mathrm{T}}$-even VV observables $\left(\left\{C_{1}[i j]\right\},\left\{C_{2}[i j]\right\},\left\{C_{13}[i j]\right\}\right)$ and $1 \mathrm{CP}$-odd and CP $\tilde{\mathrm{T}}$-even observable $\left[C_{14}[12]\right]$. However, we already saw that this latter observable vanishes. Since the $\mathrm{CP}$-violating phase $\phi_{\tau}$ in the tau slepton system affects neither the masses of the $\tilde{\tau}$ eigenstates nor the absolute values of their gauge couplings, it cannot be directly measured through VV correlations only. ${ }^{\dagger}$ However, the absolute values of $X_{\tau}, Y_{\tau}$ and $Z_{\tau}$ defined in Eq. (9) can be determined from the remaining $9 \mathrm{CP}$-even and $\mathrm{CP} \tilde{\mathrm{T}}$-even observables, together with the measurement of the two tau slepton masses. As a result, if $\tan \beta$ is determined (or constrained to be large) from other processes (e.g., chargino pair production in $e^{+} e^{-}$collisions [33]), the above determination can be used to obtain the left/right soft-breaking tau slepton masses $m_{\tilde{\tau}_{L, R}}$. The absolute magnitude of $\left|A_{\tau}^{*}+\mu \mathrm{e}^{i \xi} \tan \beta\right|$ can be determined even if $\tan \beta$ is unknown. However, it is necessary to consider different observables to determine the phase angle $\phi_{\tau}=\arg \left(-A_{\tau}-\mu^{*} \mathrm{e}^{-i \xi}\right)$.

\subsubsection{Scalar-scalar correlations}

The scalar-scalar correlations involve not only tau slepton mixing but also neutral Higgs boson mixing. However, it is known that the Higgs boson mixing angles can be completely determined through on-shell production of each Higgs boson with polarized muons [21]. In this section we therefore check the possibility of reconstructing the tau slepton system through these scalarscalar correlations. There are 16 such correlations: $7 \mathrm{CP}$-even and $\mathrm{CP} \tilde{\mathrm{T}}-$ even, $1 \mathrm{CP}$-even and CP $\tilde{\mathrm{T}}$-odd, $3 \mathrm{CP}$-odd and CP $\tilde{\mathrm{T}}$-even and $5 \mathrm{CP}$-odd and CP $\tilde{\mathrm{T}}$-odd observables. It is straightforward to obtain the explicit forms of the these 16 correlations:

$$
\begin{aligned}
& \left\{C_{3}[i j]\right\}=+\left(\frac{Y_{\mu}}{c_{W}^{2} s_{W}^{2}}\right)^{2} \frac{m_{W}^{2}}{2 s} \mathcal{S}_{k l} \Re \mathrm{e}\left(V_{k ; i j} V_{l ; i j}^{*}\right)\left(O_{2 k} O_{2 l}+s_{\beta}^{2} O_{1 k} O_{1 l}\right), \\
& {\left[C_{3}[12]\right]=-\left(\frac{Y_{\mu}}{c_{W}^{2} s_{W}^{2}}\right)^{2} \frac{m_{W}^{2}}{2 s} \mathcal{D}_{k l} \Im \mathrm{m}\left(V_{k ; 12} V_{l ; 12}^{*}\right)\left(O_{2 k} O_{2 l}+s_{\beta}^{2} O_{1 k} O_{1 l}\right),} \\
& \left\{C_{4}[i j]\right\}=+\left(\frac{Y_{\mu}}{c_{W}^{2} s_{W}^{2}}\right)^{2} \frac{m_{W}^{2}}{2 s} \mathcal{D}_{k l} \Re \mathrm{e}\left(V_{k ; i j} V_{l ; i j}^{*}\right) s_{\beta}\left(O_{1 k} O_{2 l}-O_{2 k} O_{1 l}\right), \\
& {\left[C_{4}[12]\right]=+\left(\frac{Y_{\mu}}{c_{W}^{2} s_{W}^{2}}\right)^{2} \frac{m_{W}^{2}}{2 s} \mathcal{S}_{k l} \Im \mathrm{m}\left(V_{k ; 12} V_{l ; 12}^{*}\right) s_{\beta}\left(O_{1 k} O_{2 l}-O_{2 k} O_{1 l}\right),} \\
& \left\{C_{15}[i j]\right\}=+\left(\frac{Y_{\mu}}{c_{W}^{2} s_{W}^{2}}\right)^{2} \frac{m_{W}^{2}}{2 s} \mathcal{S}_{k l} \Re \mathrm{e}\left(V_{k ; i j} V_{l ; i j}^{*}\right)\left(O_{2 k} O_{2 l}-s_{\beta}^{2} O_{1 k} O_{1 l}\right),
\end{aligned}
$$

\footnotetext{
${ }^{\dagger}$ The phase $\Phi_{A_{\tau}}$ does affect the $\tilde{\tau}$ masses. However, it could be extracted from measurements of $\tilde{\tau}$ masses and $\theta_{\tau}$ only if $\left|A_{\tau}\right|$ and $|\mu| \tan \beta$ are already known. In case of $\left|A_{\tau}\right|$ at least this seems quite unlikely.

${ }^{\ddagger}$ The absolute value of $Z_{\tau}$ does depend on the relative phase between $A_{\tau}^{*}$ and $\mu \mathrm{e}^{i \xi}$. However, observables that only depend on $\left|Z_{\tau}\right|$ clearly cannot determine this phase, unless $\left|A_{\tau}\right|$ and $|\mu \tan \beta|$ are already known.
} 


$$
\begin{aligned}
& {\left[C_{15}[12]\right]=-\left(\frac{Y_{\mu}}{c_{W}^{2} s_{W}^{2}}\right)^{2} \frac{m_{W}^{2}}{2 s} \mathcal{D}_{k l} \Im \mathrm{m}\left(V_{k ; 12} V_{l ; 12}^{*}\right)\left(O_{2 k} O_{2 l}-s_{\beta}^{2} O_{1 k} O_{1 l}\right),} \\
& \left\{C_{16}[i j]\right\}=-\left(\frac{Y_{\mu}}{c_{W}^{2} s_{W}^{2}}\right)^{2} \frac{m_{W}^{2}}{2 s} \mathcal{S}_{k l} \Re \mathrm{e}\left(V_{k ; i j} V_{l ; i j}^{*}\right) s_{\beta}\left(O_{1 k} O_{2 l}+O_{2 k} O_{1 l}\right), \\
& {\left[C_{16}[12]\right]=+\left(\frac{Y_{\mu}}{c_{W}^{2} s_{W}^{2}}\right)^{2} \frac{m_{W}^{2}}{2 s} \mathcal{D}_{k l} \Im \mathrm{m}\left(V_{k ; 12} V_{l ; 12}^{*}\right) s_{\beta}\left(O_{1 k} O_{2 l}+O_{2 k} O_{1 l}\right),}
\end{aligned}
$$

where summation over the repeating indices $k$ and $l$ is understood. In deriving Eqs. (44) we have exploited the relation $V_{k ; i j}=\left(V_{k ; j i}\right)^{*}$. Note that any rate asymmetry, defined to be proportional to the difference between the [12] and [21] modes, is determined by the combination $\Im \mathrm{m}\left(V_{k ; 12} V_{l: 12}^{*}\right)$, which is antisymmetric with respect to the Higgs boson indices $k, l$. In addition, in the MSSM the lightest Higgs boson has a mass of less than $130 \mathrm{GeV}$ and a tiny width, so that its contribution to the tau slepton pair production is negligible. As a result, only the propagator combinations $\mathcal{S}_{22}, \mathcal{S}_{23}, \mathcal{S}_{33}$ and $\mathcal{D}_{23}$ are important in determining the $\sqrt{s}$ dependence of these rate asymmetries.

\subsubsection{Scalar-vector correlations}

All the SV correlations are proportional to the real or imaginary part of a Higgs propagator, so that they also are sizable when the c.m. energy is very close to one of the heavy Higgs boson resonances. ${ }^{\S}$

$$
\begin{aligned}
& \left\{C_{5}[i j]\right\}=+\frac{m_{W} Y_{\mu} \beta}{4 \sqrt{s} c_{W}^{4} s_{W}^{4}} O_{2 k} D_{Z} \Re \mathrm{e}\left[D_{H_{k}}\right] \Re \mathrm{e}\left(V_{k ; i j} Q_{i j}^{Z *}\right) \sin \Theta, \\
& {\left[C_{5}[12]\right]=-\frac{m_{W} Y_{\mu} \beta}{4 \sqrt{s} c_{W}^{4} s_{W}^{4}} O_{2 k} D_{Z} \Im \mathrm{m}\left[D_{H_{k}}\right] \Im \mathrm{m}\left(V_{k ; 12} Q_{12}^{Z *}\right) \sin \Theta,} \\
& \left\{C_{6}[i j]\right\}=-\frac{m_{W} Y_{\mu} \beta}{\sqrt{s} c_{W}^{2} s_{W}^{2}} O_{2 k} \Im \mathrm{m}\left[D_{H_{k}}\right] \Re \mathrm{e}\left(V_{k ; i j} \delta_{i j}+D_{Z} \frac{s_{W}^{2}-1 / 4}{c_{W}^{2} s_{W}^{2}} V_{k ; i j} Q_{i j}^{Z *}\right) \sin \Theta, \\
& {\left[C_{6}[12]\right]=-\frac{m_{W} Y_{\mu} \beta}{\sqrt{s} c_{W}^{4} s_{W}^{4}}\left(s_{W}^{2}-1 / 4\right) O_{2 k} D_{Z} \Re \mathrm{e}\left[D_{H_{k}}\right] \Im \mathrm{m}\left(V_{k ; 12} Q_{12}^{Z *}\right) \sin \Theta,} \\
& \left\{C_{7}[i j]\right\}=-\frac{m_{W} Y_{\mu} \beta}{\sqrt{s} c_{W}^{2} s_{W}^{2}} s_{\beta} O_{1 k} \Im \mathrm{m}\left[D_{H_{k}}\right] \Re \mathrm{e}\left(V_{k ; i j} \delta_{i j}+D_{Z} \frac{s_{W}^{2}-1 / 4}{c_{W}^{2} s_{W}^{2}} V_{k ; i j} Q_{i j}^{Z *}\right) \sin \Theta, \\
& {\left[C_{7}[12]\right]=-\frac{m_{W} Y_{\mu} \beta}{\sqrt{s} c_{W}^{4} s_{W}^{4}}\left(s_{W}^{2}-1 / 4\right) s_{\beta} O_{1 k} D_{Z} \Re \mathrm{e}\left[D_{H_{k}}\right] \Im \mathrm{m}\left(V_{k ; 12} Q_{12}^{Z *}\right) \sin \Theta,} \\
& \left\{C_{8}[i j]\right\}=-\frac{m_{W} Y_{\mu} \beta}{4 \sqrt{s} c_{W}^{4} s_{W}^{4}} s_{\beta} O_{1 k} D_{Z} \Re \mathrm{e}\left[D_{H_{k}}\right] \Re \mathrm{e}\left(V_{k ; i j} Q_{i j}^{Z *}\right) \sin \Theta, \\
& {\left[C_{8}[12]\right]=+\frac{m_{W} Y_{\mu} \beta}{4 \sqrt{s} c_{W}^{4} s_{W}^{4}} s_{\beta} O_{1 k} D_{Z} \Im \mathrm{m}\left[D_{H_{k}}\right] \Im \mathrm{m}\left(V_{k ; 12} Q_{12}^{Z *}\right) \sin \Theta,}
\end{aligned}
$$

$\S \Re \mathrm{e}\left(D_{H_{k}}\right)=0$ for $\sqrt{s}=m_{H_{k}}$. However, since the mass splitting between the two heavy Higgs states is small, the contribution from the second heavy Higgs boson is usually still sizable at this point. 


$$
\begin{aligned}
& \left\{C_{9}[i j]\right\}=+\frac{m_{W} Y_{\mu} \beta}{4 \sqrt{s} c_{W}^{4} s_{W}^{4}} s_{\beta} O_{1 k} D_{Z} \Im \mathrm{m}\left[D_{H_{k}}\right] \Re \mathrm{e}\left(V_{k ; i j} Q_{i j}^{Z *}\right) \sin \Theta \\
& {\left[C_{9}[12]\right]=+\frac{m_{W} Y_{\mu} \beta}{4 \sqrt{s} c_{W}^{4} s_{W}^{4}} s_{\beta} O_{1 k} D_{Z} \Re \mathrm{e}\left[D_{H_{k}}\right] \Im \mathrm{m}\left(V_{k ; 12} Q_{12}^{Z *}\right) \sin \Theta} \\
& \left\{C_{10}[i j]\right\}=+\frac{m_{W} Y_{\mu} \beta}{\sqrt{s} c_{W}^{2} s_{W}^{2}} s_{\beta} O_{1 k} \Re \mathrm{e}\left[D_{H_{k}}\right] \Re \mathrm{e}\left(V_{k ; i j} \delta_{i j}+D_{Z} \frac{s_{W}^{2}-1 / 4}{c_{W}^{2} s_{W}^{2}} V_{k ; i j} Q_{i j}^{Z *}\right) \sin \Theta, \\
& {\left[C_{10}[12]\right]=-\frac{m_{W} Y_{\mu} \beta}{\sqrt{s} c_{W}^{4} s_{W}^{4}}\left(s_{W}^{2}-1 / 4\right) s_{\beta} O_{1 k} D_{Z} \Im \mathrm{m}\left[D_{H_{k}}\right] \Im \mathrm{m}\left(V_{k ; 12} Q_{12}^{Z *}\right) \sin \Theta} \\
& \left\{C_{11}[i j]\right\}=+\frac{m_{W} Y_{\mu} \beta}{\sqrt{s} c_{W}^{2} s_{W}^{2}} O_{2 k} \Re \mathrm{e}\left[D_{H_{k}}\right] \Re \mathrm{e}\left(V_{k ; i j} \delta_{i j}+D_{Z} \frac{s_{W}^{2}-1 / 4}{c_{W}^{2} s_{W}^{2}} V_{k ; i j} Q_{i j}^{Z *}\right) \sin \Theta, \\
& {\left[C_{11}[12]\right]=-\frac{m_{W} Y_{\mu} \beta}{\sqrt{s} c_{W}^{4} s_{W}^{4}}\left(s_{W}^{2}-1 / 4\right) O_{2 k} D_{Z} \Im \mathrm{m}\left[D_{H_{k}}\right] \Im \mathrm{m}\left(V_{k ; 12} Q_{i j}^{Z *}\right) \sin \Theta,} \\
& \left\{C_{12}[i j]\right\}=-\frac{m_{W} Y_{\mu}^{\beta}}{4 \sqrt{s} c_{W}^{4} s_{W}^{4}} O_{2 k} D_{Z} \Im \mathrm{m}\left[D_{H_{k}}\right] \Re \mathrm{e}\left(V_{k ; i j} Q_{i j}^{Z *}\right) \sin \Theta \\
& {\left[C_{12}[12]\right]=-\frac{m_{W} Y_{\mu} \beta}{4 \sqrt{s} c_{W}^{4} s_{W}^{4}} O_{2 k} D_{Z} \Re \mathrm{e}\left[D_{H_{k}}\right] \Im \mathrm{m}\left(V_{k ; 12} Q_{i j}^{Z *}\right) \sin \Theta .}
\end{aligned}
$$

The CP and CP T properties of the observables in Eq. (45) can be identified by noting the following aspects: (i) observables involving $\Re \mathrm{e}\left[D_{H_{k}}\right]\left(\Im \mathrm{m}\left[D_{H_{k}}\right]\right)$ are CP $\tilde{\mathrm{T}}-$ even $(\mathrm{CP} \tilde{\mathrm{T}}$-odd). (ii) The matrix elements $O_{2 k}$ and $O_{1 k}$ are the $\mathrm{CP}$-even and $\mathrm{CP}$-odd component of the neutral Higgs boson $H_{k}$, respectively. (iii) $\Re \mathrm{e}\left(V_{k ; i j} \delta_{i j}\right)$ and $\Re \mathrm{e}\left(V_{k ; i j} Q_{i j}^{Z *}\right)$ are $\mathrm{CP}$-even, while $\Im \mathrm{m}\left(V_{k ; 12} Q_{12}^{Z *}\right)$ is $\mathrm{CP}$-odd. For instance, $\left\{C_{5}[i j]\right\}$ is $\mathrm{CP}$-even and $\mathrm{CP} \tilde{\mathrm{T}}$-even, while $\left[C_{6}[12]\right]$ is $\mathrm{CP}$-odd and $\mathrm{CP} \tilde{\mathrm{T}}-$ even. Of course, this determination of the $\mathrm{CP}$ and $\mathrm{CP} \tilde{\mathrm{T}}$ properties agrees with the results of Table 2.

\subsection{Extracting the polarization and rate asymmetries}

In this section we investigate efficient procedures for extracting the relevant SUSY parameters from the polarized cross sections. In actual experiments, a careful analysis of statistical and systematic uncertainties will be required when extracting the rate and polarization asymmetries. We do not attempt to estimate realistic uncertainties based on event simulation or parameter fitting. Instead, we are interested in the question which of the numerous quantities listed in the previous Subsection are most sensitive to the fundamental parameters of the theory, our main focus being on $\mathrm{CP}$-odd phases in the interaction Lagrangian.

The conceptually simplest asymmetry is the total unpolarized rate asymmetry, defined by

$$
A_{R}^{\text {tot }}=\frac{\int d \cos \Theta\left(\left[C_{1}[12]\right]+\left[C_{3}[12]\right]\right)}{\int d \cos \Theta\left(\left\{C_{1}[12]\right\}+\left\{C_{3}[12]\right\}\right)} .
$$

Unfortunately this asymmetry is usually very small even if $\mathrm{CP}$ is violated in both the Higgs and $\tilde{\tau}$ sector. We saw in Eq. (40) that $\left[C_{1}[12]\right]$ vanishes in the limit $\Gamma_{Z} \rightarrow 0$. Moreover, 
Eq. (44) shows that $\left[C_{3}[12]\right]$ is proportional to $O_{2 k} O_{2 l}+\sin ^{2} \beta O_{1 k} O_{1 l}$. The contribution from Higgs exchange can only compete with the gauge boson exchange contributions if $\sqrt{s} \simeq m_{\text {Higgs }}$. Given LEP search limits for $\tilde{\tau}_{1}$, real $\tilde{\tau}$ pair production will only be possible at energies $s \gg m_{Z}^{2} \cdot$ ฯ These two requirements together imply that we need $m_{A}^{2} \gg m_{Z}^{2}$. In this "decoupling limit" the lightest neutral Higgs boson eigenstate is given by $H_{1} \simeq \cos \beta \phi_{1}+\sin \beta \phi_{2}$; the couplings of this state are very similar to that of the Higgs boson of the Standard Model. The rotation matrix $O$ of Eq. (7) is then approximately given by:

$$
O \simeq\left(\begin{array}{ccc}
0 & \cos \alpha_{H} & \sin \alpha_{H} \\
\cos \beta & \sin \alpha_{H} \sin \beta & -\cos \alpha_{H} \sin \beta \\
\sin \beta & -\sin \alpha_{H} \cos \beta & \cos \alpha_{H} \cos \beta
\end{array}\right)
$$

where $\sin \left(2 \alpha_{H}\right) \neq 0$ signals $\mathrm{CP}$-violation in the Higgs sector. The value of $\alpha_{H}$ depends in a complicated manner on the parameters appearing in the squark mass matrix, and on $m_{A}$. However, the structure of the rotation matrix (47) implies that

$$
O_{2 k} O_{2 l}+\sin ^{2} \beta O_{1 k} O_{1 l} \simeq \sin ^{2} \beta \delta_{l k}, \quad l, k \in 2,3 .
$$

On the other hand, the factor $\mathcal{D}_{k l}$ appearing in the expression for $\left[C_{3}[12]\right]$ is nonzero only for $k \neq l$. These two requirements are incompatible, i.e. the total rate asymmetry is strongly suppressed.

We thus have to make use of asymmetries that are sensitive to the beam polarization. To this end we introduce rate and polarization asymmetries with respect to the unpolarized vector boson exchange and Higgs boson exchange parts, $\left\{C_{1}[i j]\right\}$ and $\left\{C_{3}[i j]\right\}$, respectively, as follows:

$$
\begin{aligned}
& \mathcal{A}_{P}\left(C_{2}[i j]\right)=\Omega_{2} \frac{\int d \cos \Theta\left\{C_{2}[i j]\right\}}{\int d \cos \Theta\left\{C_{1}[i j]\right\}}, \\
& \mathcal{A}_{R}\left(C_{2}[12]\right)=\Omega_{2} \frac{\int d \cos \Theta\left[C_{2}[12]\right]}{\int d \cos \Theta\left\{C_{1}[12]\right\}}, \\
& \mathcal{A}_{P}\left(C_{4}[i j]\right)=\Omega_{4} \frac{\int d \cos \Theta\left\{C_{4}[i j]\right\}}{\int d \cos \Theta\left\{C_{3}[i j]\right\}}, \\
& \mathcal{A}_{R}\left(C_{4}[12]\right)=\Omega_{4} \frac{\int d \cos \Theta\left[C_{4}[12]\right]}{\int d \cos \Theta\left\{C_{3}[12]\right\}} .
\end{aligned}
$$

The remaining $12 \times 4$ correlations $(n=5-16)$ can only be measured if at least one of the initial beams is transversely polarized. In this case the proper normalization involves the sum of the unpolarized Higgs and gauge boson exchange contributions:

$$
\mathcal{A}_{P}\left(C_{n}[i j]\right)=\Omega_{n} \frac{\int d \cos \Theta\left\{C_{n}[i j]\right\}}{\int d \cos \Theta\left(\left\{C_{1}[i j]\right\}+\left\{C_{3}[i j]\right\}\right)},
$$

\footnotetext{
This also implies that in the MSSM the lightest Higgs boson mass must be smaller than the mass of the lightest tau slepton pair, so that its contribution to any $\tilde{\tau}$ pair production process is negligible.

"This corrects an erroneous numerical result in Ref. [17.
} 


$$
\mathcal{A}_{R}\left(C_{n}[12]\right)=\Omega_{n} \frac{\int d \cos \Theta\left[C_{n}[12]\right]}{\int d \cos \Theta\left(\left\{C_{1}[12]\right\}+\left\{C_{3}[12]\right\}\right)} .
$$

The $\Omega_{n}$ in Eqs. (49) and (50) are numerical factors that originate from projecting out the corresponding observable, by adjusting the (anti-)muon polarization and integrating the distribution $\Sigma_{i j}$ over the azimuthal angle $\Phi$ with an appropriate weight function, as described below.

These factors $\Omega_{n}(n=2,4,5-16)$ depend sensitively on the degrees of longitudinal and transverse polarizations of the muon and anti-muon beams achievable at muon colliders. For the sake of discussion, let us assume that both the muon and anti-muon beams are polarized with perfect degrees of longitudinal or transverse polarization. From Eq. (33) we can then deduce the algorithms for extracting our asymmetries, which determine the $\Omega_{n}$. In the following discussion we always assume that all cross sections are appropriately (anti-)symmetrized in the $\tilde{\tau}$ indices.

- The asymmetries $\propto C_{2}[i j]$ can be measured by dividing the difference of cross sections for $P_{L}=-\bar{P}_{L}=1$ and $P_{L}=-\bar{P}_{L}=-1$ by the sum of these cross sections; this gives $\Omega_{2}=1$. . N Note that a CP-transformation leaves the polarization states used in this prescription invariant. The asymmetries $\propto C_{4}[i j]$ can be obtained in the same way from the cross sections for $P_{L}=\bar{P}_{L}=1$ and $P_{L}=\bar{P}_{L}=-1$, so that $\Omega_{4}=1$ as well. In this case a CP transformation maps the two polarization states into each other; this difference of cross sections is therefore a CP-odd quantity, in accord with the results of Table 1.

- The asymmetries involving $C_{15}$ and $C_{16}$ can be obtained by fixing $P_{T}=\bar{P}_{T}=1$ and taking the ratio of the difference and the sum of the distributions for $\cos (\alpha-\bar{\alpha})= \pm 1$ $(\sin (\alpha-\bar{\alpha})= \pm 1)$, respectively. (Recall that $P_{T}=\bar{P}_{T}=1$ implies $P_{L}=\bar{P}_{L}=0$, and that $\alpha-\bar{\alpha}$ is independent of $\Phi$.) This gives $\Omega_{15}=\Omega_{16}=1$. Recall that a CP transformation flips the sign of $\alpha-\bar{\alpha}$. Since the cosine is invariant under the change of sign of its argument while the sine changes its sign, the prescription for extracting $C_{15}$ is $\mathrm{CP}$-even while that for $C_{16}$ is $\mathrm{CP}$-odd, again in accordance with the $\mathrm{CP}$ transformation properties of the corresponding terms in the squared matrix element; see Table 1.

- The asymmetries involving $C_{13}$ and $C_{14}$ (which vanishes in the MSSM in the limit $\Gamma_{Z}=0$ ) can also be obtained by chosing $P_{T}=\bar{P}_{T}=1$. However, the angular combinations $\cos (\alpha+\bar{\alpha})(\sin (\alpha+\bar{\alpha}))$ depend on the azimuthal angle $\Phi$, see Eq. (36). Therefore we need to apply a (CP-even) normalized weight function $\sqrt{2} \cos (\alpha+\bar{\alpha})(\sqrt{2} \sin (\alpha+\bar{\alpha}))$ in order to extract these asymmetries, where $\alpha+\bar{\alpha}=\eta-2 \Phi$. The normalization factors in these weight functions $f(\Phi)$ are determined by the requirement that $\int d \Phi f(\Phi)$ acts as a projector, which implies $\int_{0}^{2 \pi} d \Phi f^{2}(\Phi)=1$. Note that this weight factor should not be applied in the denominator of Eq. (50). Finally, one has to take the sum of these weighted integrated cross sections for $\eta=0$ and $\eta=\pi$, in order to remove terms $\propto C_{15}$ or $C_{16}$ from the denominator 5 ; note that these two configurations of transverse polarization vectors are self-conjugate under a CP transformation, since $\eta=-\pi$ is identical to $\eta=+\pi$. We

${ }^{* *}$ Recall that $\left[C_{2}[12]\right]=0$ in the MSSM if the $\mathrm{Z}$ width is neglected.

${ }^{\dagger \dagger}$ The numerator is independent of $\eta$ after the weighted integration over $\Phi$. 
thus find $\Omega_{13}=\Omega_{14}=1 / \sqrt{2}$. Since $\alpha+\bar{\alpha}$ is also invariant under CP-transformations this extraction prescription is $\mathrm{CP}$-even, in accord with the corresponding entries in Table 1.

- The asymmetries $\propto C_{n}(n=5-8)$ are obtained from the cross sections for $P_{T}=$ 1, $\bar{P}_{T}=\bar{P}_{L}=0$ and $\bar{P}_{T}=1, P_{T}=P_{L}=0$, taking $\eta=0 . C_{5}$ and $C_{6}$ can be obtained from the $\left(\mathrm{CP}\right.$-even) sum of the corresponding cross sections, while $C_{7}$ and $C_{8}$ can be determined from the ( $\mathrm{CP}$-odd) difference of cross sections. In addition one needs the weight function $\sqrt{2} \cos \alpha$ for $C_{5}$ and $C_{7}$, and $\sqrt{2} \sin \alpha$ for $C_{6}$ and $C_{8}$; for $\eta=0$ these weight functions are all $\mathrm{CP}$-even, so that the extraction procedure again has the same CP property as the corresponding coefficient of the squared matrix element. This yields $\Omega_{5}=\Omega_{6}=\Omega_{7}=\Omega_{8}=1 / \sqrt{2}$.

- The algorithm for extracting the asymmetries $\propto C_{n}(n=9-12)$ is complicated. One possible procedure is to measure the distributions for the polarization combinations $\left\{P_{L}=\right.$ $\left.\pm 1, \bar{P}_{T}=1\right\}$ and $\left\{\bar{P}_{L}= \pm 1, P_{T}=1\right\}$ for $\eta=0$. Denote these four distributions by $\Sigma_{ \pm}^{(1)}$ and $\Sigma_{ \pm}^{(2)}$. A CP-transformation then sends $\Sigma_{ \pm}^{(1)}$ to $\Sigma_{\mp}^{(2)}$ and vice versa. The asymmetries $\propto C_{9}$ and $C_{11}$ can then be extracted from $\Sigma_{+}^{(1)}-\Sigma_{-}^{(1)} \pm\left(\Sigma_{+}^{(2)}-\Sigma_{-}^{(2)}\right)$, using the weight function $\sqrt{2} \cos \alpha$; note that the plus (minus) sign in front of the round parentheses gives a $\mathrm{CP}$-odd ( $\mathrm{CP}$-even) combination of cross sections. The asymmetries $\propto C_{10}$ and $\propto C_{12}$ can be extracted in the same way, if the weight function $\sqrt{2} \sin \alpha$ is used. This gives $\Omega_{9}=\Omega_{10}=\Omega_{11}=\Omega_{12}=1 / \sqrt{2}$.

In reality the degrees of longitudinal and transverse polarization are not perfect, in which case the polarization factors $\Omega_{n}$ should be multiplied by the relevant (products of) degrees of longitudinal and transverse polarizations, $\chi_{L}$ and $\chi_{T}\left(\bar{\chi}_{L}\right.$ and $\left.\bar{\chi}_{T}\right)$, of the incident muon (antimuon) beam. As already stated, we will simply take $\chi_{L}=\chi_{T}=\bar{\chi}_{L}=\bar{\chi}_{T}=1$ in the following numerical analysis.

\section{$5 \quad$ Numerical results}

In this section, we present some numerical analyses of CP violation both in the tau-slepton and neutral Higgs boson systems based on a specific scenario for the relevant SUSY parameters.

The loop-induced CP violation in the Higgs sector can only be large if both $|\mu|$ and $\left|A_{t}\right|$ (or $\left|A_{b}\right|$, if $\tan \beta \gg 1$ ) are sizable [12, 13, 14, 15]. For definiteness we will present results for $\tan \beta=10$ and the c.m. energy $\sqrt{s}$ near the two heavy Higgs boson resonances. Since even for $\tan \beta=10$ the contributions from the bottom (s)quark sector are still quite small, our results are not sensitive to $m_{\tilde{D}}$ and $A_{b}$; we therefore fix $A_{b}=A_{t}$ and $m_{\tilde{D}}=m_{\tilde{U}}=m_{\tilde{Q}}$, although different values for the $\mathrm{SU}(2)$ doublet and singlet soft breaking squark masses, $m_{\tilde{Q}} \neq m_{\tilde{U}}$, are allowed, and also take equal phases for $A_{t}$ and $A_{b}$. Since we are basically interested in distinguishing the $\mathrm{CP}$ non-invariant Higgs sector from the $\mathrm{CP}$ invariant one, we take for the re-phasing-invariant phase $\Phi_{A \mu}$ of $A_{t, b} \mu \mathrm{e}^{i \xi}$ two values; 0 (CP invariant case), and $\pi / 2$ (almost maximally $\mathrm{CP}$ violating case) while the phase of the gluino mass $\Phi_{\tilde{g}}$ is set to be zero. We chose 
the following "standard set" of real mass parameters and couplings of the Higgs and squark sectors:

$$
\begin{aligned}
& m_{A}=0.5 \mathrm{TeV}, \quad\left|A_{t, b}\right|=1.0 \mathrm{TeV}, \quad|\mu|=2.0 \mathrm{TeV} \\
& m_{\tilde{g}}=0.5 \mathrm{TeV}, \quad \Phi_{\tilde{g}}=0, \quad m_{\tilde{Q}}=m_{\tilde{U}}=m_{\tilde{D}}=1.0 \mathrm{TeV}
\end{aligned}
$$

In our numerical analysis, the top and bottom quark running masses $\bar{m}_{t}\left(m_{t}\right)=165 \mathrm{GeV}$ and $\bar{m}_{b}\left(m_{b}\right)=4.2 \mathrm{GeV}$ will be taken. For $m_{A}$ much larger than $m_{Z}$ as in Eq. (51), two neutral Higgs bosons have almost degenerate masses:

$$
\begin{array}{ll}
\Phi_{A \mu}=0: & m_{H_{2}}=499.6 \mathrm{GeV}, \quad m_{H_{3}}=500.0 \mathrm{GeV} \\
\Phi_{A \mu}=\frac{\pi}{2}: & m_{H_{2}}=499.5 \mathrm{GeV}, \quad m_{H_{3}}=500.4 \mathrm{GeV}
\end{array}
$$

In the CP invariant case $\left(\Phi_{A \mu}=0\right)$ we have $\cos \alpha_{H}=0$, i.e. the state $H_{2}$ is CP even and the state $H_{3}$ is $\mathrm{CP}$ odd. On the other hand, our parameter set with $\Phi_{A \mu}=\pi / 2$ gives $\cos \alpha_{H}=0.976$, i.e. now the state $\mathrm{H}_{2}$ is mostly (but not entirely) $\mathrm{CP}$-odd, and $H_{3}$ is mostly $\mathrm{CP}$-even. Because of the near-degeneracy of the two heavy Higgs bosons a significant overlap effect, i.e. interference between these two Higgs boson resonances is expected in the presence of CP violation. Note that $\mathrm{CP}$ violation in the Higgs sector tends to increase the mass splitting between the two heavy states; however, in our case it still does not exceed the widths of the two Higgs bosons, which amount to about $1.2 \mathrm{GeV}$.

We work in the general MSSM, i.e. we do not assume unification of scalar soft breaking parameters (masses and $A$-terms). The real SUSY parameters associated with the tau slepton system are thus independent of the parameters in the Higgs and squark sectors. We take as standard inputs:

$$
m_{\tilde{\tau}_{L}}=0.23 \mathrm{TeV}, \quad m_{\tilde{\tau}_{R}}=0.18 \mathrm{TeV}, \quad\left|A_{\tau}\right|=0.5 \mathrm{TeV}
$$

The phase $\Phi_{A_{\tau}}$ of the rephasing invariant quantity $A_{\tau} \mu \mathrm{e}^{i \xi}$ is varied. For reference, we list the tau slepton masses for $\Phi_{A_{\tau}}=0, \pi / 2$ :

$$
\begin{array}{lll}
\Phi_{A_{\tau}}=0: & m_{\tilde{\tau}_{1}}=82.7 \mathrm{GeV}, & m_{\tilde{\tau}_{2}}=287.4 \mathrm{GeV} \\
\Phi_{A_{\tau}}=\frac{\pi}{2}: & m_{\tilde{\tau}_{1}}=87.6 \mathrm{GeV}, & m_{\tilde{\tau}_{2}}=286.0 \mathrm{GeV}
\end{array}
$$

Since the c.m. energy is assumed to be around the resonances of the heavy Higgs bosons, $\sqrt{s} \simeq 500 \mathrm{GeV}$, the tau slepton pairs $\tilde{\tau}_{1}^{+} \tilde{\tau}_{1}^{-}$and $\tilde{\tau}_{1}^{ \pm} \tilde{\tau}_{2}^{\mp}$ can be produced in $\mu^{+} \mu^{-}$collisions, but heavy $\left(\tilde{\tau}_{2}^{+} \tilde{\tau}_{2}^{-}\right)$pairs are not accessible, as can be seen from Eq. (52).

In the previous section, we have listed 64 polarization observables which can be constructed if the production plane can be reconstructed (at least statistically), i.e. if both the polar angle $\Theta$ and the azimuthal angle $\Phi$ can be measured. We have found that there are $10 \mathrm{CP}$-violating rate

${ }^{\dagger}$ This implies that we cannot make statements about first or second generation sfermion masses. Moreover, we do not assume that the electroweak gauge symmetry is broken radiatively. 
asymmetries and $18 \mathrm{CP}$-violating polarization asymmetries. For some polarization observables, and for all rate asymmetries, it is important to experimentally identify the electric charges of the produced sfermions from their decay products. These requirements are very difficult to satisfy in the production of third generation squarks. Moreover, in most SUSY models squarks are significantly heavier than sleptons, so that squark pair production may not be possible for $\sqrt{s} \simeq m_{A}$. This is why we focus on the production of tau slepton pairs. Even here the requirement that the heavy Higgs boson masses are larger than the sum of the tau slepton masses imposes a nontrivial constraint on the accessible parameter space. Moreover, in the absence of a detailed Monte Carlo study it is not clear how well the production plane can be determined experimentally. In the following we therefore consider the cases without and with direct reconstruction of the production plane separately.

Before turning to these asymmetries we briefly discuss the behavior of the total $\tilde{\tau}_{1}^{+} \tilde{\tau}_{1}^{-}$and $\tilde{\tau}_{1}^{+} \tilde{\tau}_{2}^{-}$cross sections. These total cross sections are not only necessary to estimate the number of available events for a given integrated luminosity. Since (apart from overall prefactors) they also appear in the denominators of Eqs. (49) and (50), they also significantly affect the

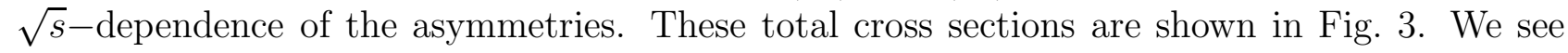
that the $\tilde{\tau}_{1}$ pair production cross section is sizable $(\sim 100 \mathrm{fb})$ even away from the Higgs poles. Since here the dominant contributions come from gauge boson exchange, there is almost no sensitivity to the $\mathrm{CP}$-violating phase $\Phi_{A_{\tau}}$, as discussed earlier. On the other hand, the Higgs exchange contribution to the total cross section does depend significantly on this phase. Not only the maximal value of this cross section, but also the energy where this maximum is reached depends on the $\mathrm{CP}$-violating phases. This follows from the observation that in the absence of $\mathrm{CP}$-violation a $\mathrm{CP}$-odd Higgs boson cannot couple to an identical $\tilde{\tau}$ pair; however, once $\mathrm{CP}$ is violated, both heavy Higgs bosons contribute. In fact, for $\Phi_{A_{\tau}} \simeq \pi$ and $\sqrt{s} \simeq m_{A}$, Higgs boson exchange clearly dominates the total $\tilde{\tau}_{1}$ pair production cross section.

The dominance of the Higgs boson exchange contributions near the poles is even more pronounced in the $\tilde{\tau}_{1} \tilde{\tau}_{2}$ channel. Our choice (53) implies $\left|\sin 2 \theta_{\tau}\right| \simeq 1$, which maximizes the $Z \tilde{\tau}_{1} \tilde{\tau}_{2}$ coupling (18). Nevertheless the gauge boson exchange contributions are suppressed compared to the case of $\tilde{\tau}_{1}$ pair production by the absence of photon exchange contributions and by the smaller available phase space $\left(\beta_{12}^{3} \simeq 0.24\right.$, as compared to $\left.\beta_{11}^{3} \simeq 0.83\right)$. Note that the Higgs boson exchange contribution only scales like $\beta$, and is hence much less phase space suppressed. Moreover, even in the absence of $\mathrm{CP}$-violation both Higgs bosons can contribute to $\tilde{\tau}_{1} \tilde{\tau}_{2}$ production. Nevertheless a strong dependence on the value of $\Phi_{A_{\tau}}$ can be seen also in this case. Note that the $\tilde{\tau}_{1} \tilde{\tau}_{2}$ production cross section typically changes by more than a factor of 10 over the shown range of energies, as compared to a variation by a factor 2 to 5 in case of $\tilde{\tau}_{1}$ pair production. The energy dependence of the total cross section is therefore especially important for the understanding of the $\sqrt{s}$ dependence of asymmetries in the $\tilde{\tau}_{1} \tilde{\tau}_{2}$ channel, which includes all rate asymmetries. Note also that the peak cross section, which falls in the range of $\sim 50$ to $\sim 300 \mathrm{fb}$, is sizable also in this channel, when compared to the anticipated luminosity of $\sim 10 \mathrm{fb}^{-1}$ per year [22]. Finally, we note that in the absence of $\mathrm{CP}$-violation

\footnotetext{
${ }_{\ddagger}^{\ddagger}$ The difference between the total cross sections for $\tilde{\tau}_{1}^{+} \tilde{\tau}_{2}^{-}$and $\tilde{\tau}_{2}^{+} \tilde{\tau}_{1}^{-}$production is negligible, as shown in Sec. 4.2 .
} 
in the Higgs sector the total cross sections depend are even functions of the phase $\Phi_{A_{\tau}}$. This explains why the curves for $\Phi_{A_{\tau}}=\phi$ coincide with those for $\Phi_{A_{\tau}}=2 \pi-\phi$ in Figs. 3(a) and (c). However, this degeneracy is lifted if $\Phi_{A \mu} \neq 0$.

(a) $\sigma_{11}, \Phi_{A_{\mu}}=0$

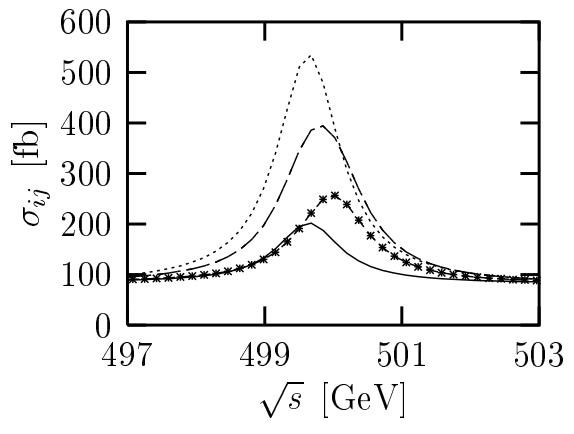

(c) $\sigma_{12}, \Phi_{A_{\mu}}=0$

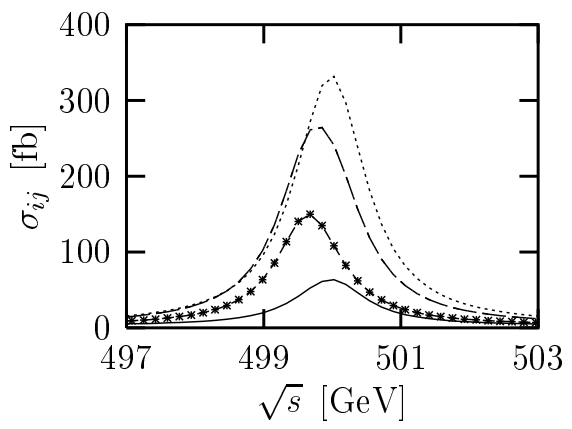

(b) $\sigma_{11}, \Phi_{A \mu}=\frac{\pi}{2}$

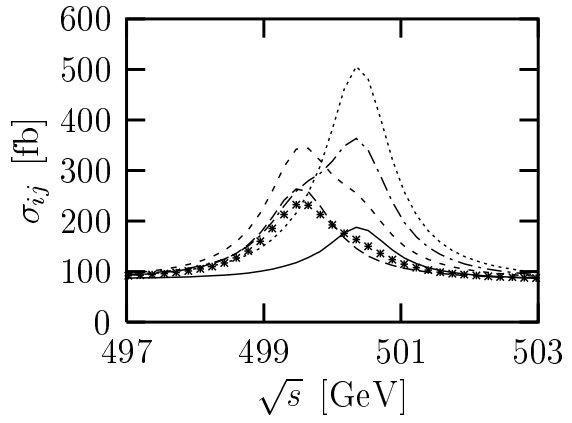

(d) $\sigma_{12}, \Phi_{A \mu}=\frac{\pi}{2}$

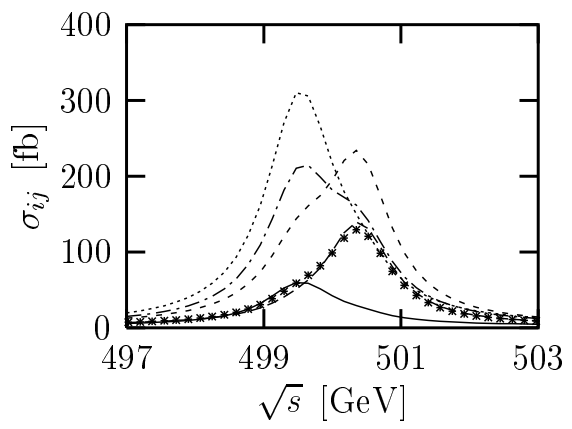

Figure 3: The $\sqrt{s}$ dependence of the total $\tilde{\tau}_{1}$ pair cross section $(a, b)$ and the mixed $\tilde{\tau}_{1}^{+} \tilde{\tau}_{2}^{-}$cross section (c,d) near the heavy Higgs boson resonances, with the SUSY parameter set of Eqs. (51), (59) and (53). Frames (a) and (c) are for $\Phi_{A \mu}=0$, while frames (b) and (d) are for $\Phi_{A \mu}=\pi / 2$, The six lines in each frame are for $\Phi_{A_{\tau}}=0$ (solid), $\pi / 3$ (long dashed), $2 \pi / 3$ (dot-dashed), $\pi$ (dotted), $4 \pi / 3$ (short dashed) and $5 \pi / 3$ (heavily dotted), respectively.

\subsection{Without direct reconstruction of the production plane}

If the production plane is not reconstructed, all the SV correlations are averaged away when integrating over the azimuthal angle $\Phi$, but the polarization and rate asymmetries corresponding to the coefficients $\left\{C_{1}, C_{2} ; C_{3}, C_{4}, C_{15}, C_{16}\right\}$ survive. As emphasized earlier, the VV correlations are, however, determined by the SUSY parameters associated with the tau slepton system without any $\mathrm{CP}$-preserving phases, if the $Z$ boson width is neglected. In this case the CP $\tilde{\mathrm{T}}-$ odd VV rate asymmetries $\mathcal{A}_{R}\left(C_{1,2}[12]\right)$ vanish so that there exist no non-trivial CP-violating VV asymmetries for probing the CP phases in the tau slepton mass matrix. This immediately

*From now on, let us denote the asymmetries with SS, VV and SV correlations in the numerators by SS, $\mathrm{VV}$ and SV asymmetries, respectively. 
implies that $\tilde{\tau}$ pair production in $e^{+} e^{-}$collisions is of no use for directly measuring the CP phase associated with the tau slepton system.I

On the other hand, the SS polarization and rate asymmetries are nonzero. The $6 \mathrm{CP}-$ violating SS polarization asymmetries $\mathcal{A}_{P}\left(C_{4}[i j]\right)$ and $\mathcal{A}_{P}\left(C_{16}[i j]\right)$ depend on $\mathrm{CP}$ violation in the $\tilde{\tau}$ sector only through the $\mathrm{CP}$-even combination of couplings $\Re \mathrm{e}\left(V_{k ; i j} V_{l ; i j}^{*}\right)$, see Eq. (44); these asymmetries are $\mathrm{CP}$-odd, since they are also proportional to the $\mathrm{CP}$-odd combinations $O_{1 k} O_{2 l} \mp O_{2 k} O_{1 l}$ of Higgs mixing angles. The product of this combination of Higgs $-\tilde{\tau} \tilde{\tau}$ couplings and Higgs mixing angles vanishes if $\mathrm{CP}$ is conserved, but in general one can expect a nonzero result in the presence of $\mathrm{CP}$ violation in either the Higgs or $\tilde{\tau}$ sector. If there is no $\mathrm{CP}-$ violation in the Higgs sector $\left(\Phi_{A \mu}=0\right.$ ), these (and all other $\mathrm{CP}$-violating) asymmetries are odd functions of $\sin \Phi_{A_{\tau}}$, i.e. the transformation $\Phi_{A_{\tau}} \rightarrow 2 \pi-\Phi_{A_{\tau}}$ leads to a change of sign of these asymmetries, leaving their absolute values unchanged. Since these are polarization asymmetries, they should be nonzero even for the production of two equal $\tilde{\tau}$ states, in particular for $\tilde{\tau}_{1}$ pair production.

These expectations are borne out by the numerical results shown in Fig. 国. Here the upper two frames show $A_{P}\left(C_{4}[11]\right)$ and the lower two frames show $A_{P}\left(C_{16}[11]\right)$, while the left (right) frames are for a $\mathrm{CP}$-conserving Higgs sector $\left(\Phi_{A \mu}=\pi / 2\right)$. Since $A_{P}\left(C_{4}[11]\right)$ is $\mathrm{CPT}$-odd, it is proportional to the imaginary part of the product of relevant Higgs boson propagators, while $A_{P}\left(C_{16}\right)$, being CP $\tilde{\mathrm{T}}$-even, is proportional to the real part of the product of propagators. Eq. (42) then explains why $A_{P}\left(C_{4}[11]\right)$ decreases much faster as one moves away from the Higgs poles than $A_{P}\left(C_{16}[11]\right)$ does. The results of Fig. 14 show that both of these polarization asymmetries are quite sensitive to $A_{\tau}$. Also, both asymmetries can be large. Finally, we note the curious fact that in the presence of sizable $\mathrm{CP}$-violation in both the Higgs and $\tilde{\tau}$ sectors some CP-odd asymmetries can be "accidentally" suppressed; see the long dashed curve (for $\left.\Phi_{A_{\tau}}=\pi / 3\right)$ in Fig. 团(b).

The possibly large size of $A_{P}\left(C_{4}[11]\right)$ is very encouraging, since this asymmetry can be measured with only longitudinally polarized beams; this is important, since it might be technically quite difficult to produce transversely polarized muon beams. Moreover, this asymmetry is linear in polarization; this means that the precision with which it can be measured also depends "only" linearly on the achievable degree of polarization. Moreover, in the presence of large $\mathrm{CP}$-violating phases this asymmetry remains sizable over a large region of parameter space. This is shown in Fig. 5, which shows the behaviour of the maximal value of $\mathcal{A}_{P}\left(C_{4}[11]\right)$ when one input parameter is moved away from our standard input set described at the beginning of this Section. The maximum here refers to the dependence on $\sqrt{s}$, i.e. we plot the asymmetry at the optimal center-of-mass energy, which depends (slightly) on the input parameters. This is legitimate, since $\sqrt{s}$ can be freely chosen in an experiment. In order to show the dependence on different parameters within a single frame, we chose as $x$-axis the ratio of a given parameter to its "standard" value. The range of variation of these parameters is restricted by experimental constraints, the most important ones being the lower bounds $m_{H_{1}} \geq 111 \mathrm{GeV}$ (note that $H_{1}$ behaves essentially like the Standard Model Higgs boson, since $\left.m_{A}^{2} \gg M_{Z}^{2}\right)$ and $m_{\tilde{\tau}_{1}} \geq 80 \mathrm{GeV}$.

\footnotetext{
${ }^{\dagger}$ An exception to this rule can occur if the two $\tilde{\tau}$ mass eigenstates happen to be nearly degenerate, with mass splitting comparable to their decay widths [29].
} 

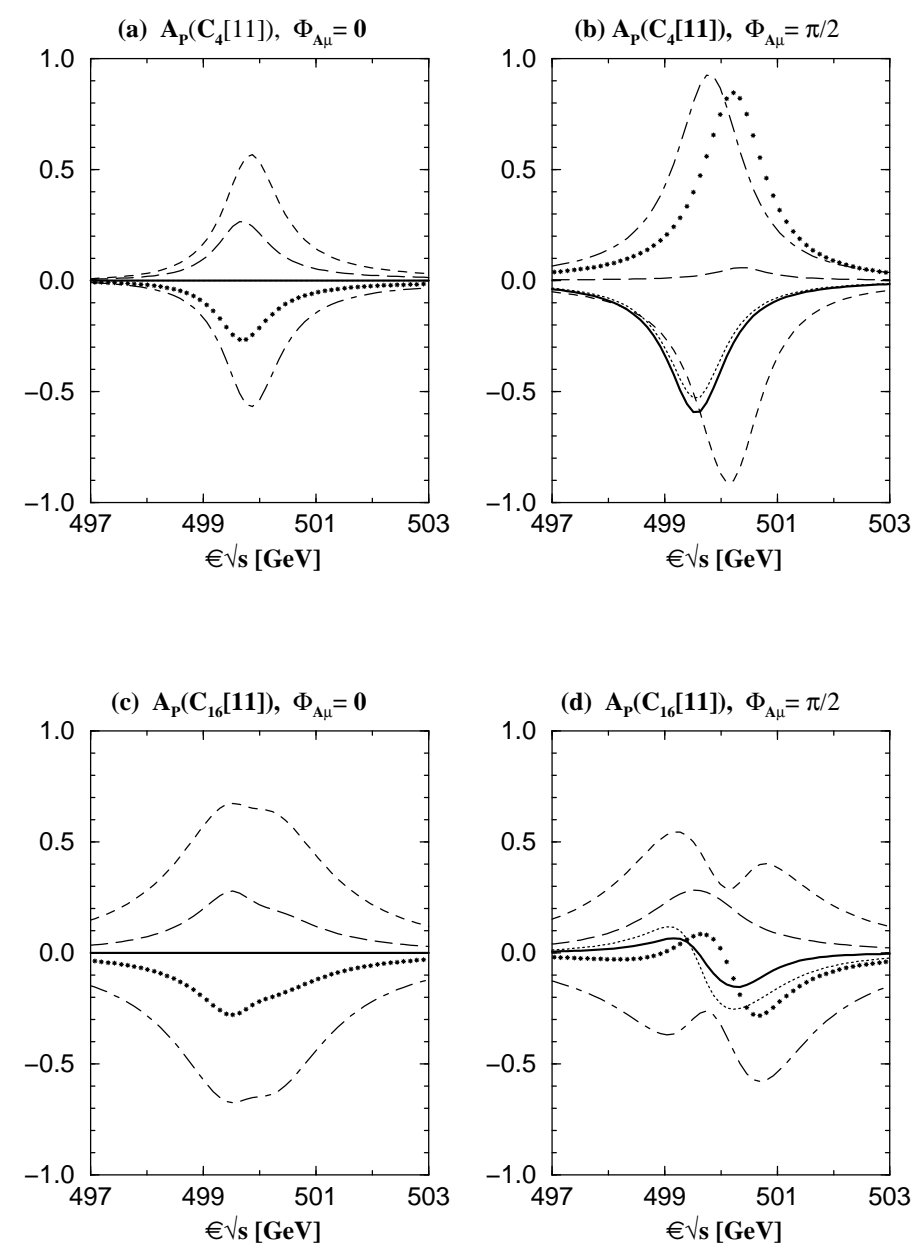

Figure 4: The $\sqrt{s}$ dependence of the $C P$-odd $S S$ polarization asymmetry $\mathcal{A}_{P}\left(C_{4}[11]\right)$ for (a) $\Phi_{A \mu}=0$ and (b) $\Phi_{A \mu}=\pi / 2$, and the $C P$-odd $S S$ polarization asymmetry $\mathcal{A}_{P}\left(C_{16}[11]\right)$ for (c) $\Phi_{A \mu}=0$ and (d) $\Phi_{A \mu}=\pi / 2$. Parameters and notation are as in Fig. 3.

We see that the maximal asymmetry depends only rather weakly on $\tan \beta$ (dashed curve). However, the total cross section near the Higgs resonance falls $\propto \tan ^{4} \beta$ for $\tan ^{2} \beta \ll m_{t} / m_{b}$, due to the decrease of the couplings of the heavy Higgs bosons to $\tilde{\tau}$ sleptons and the simultaneous increase of the Higgs decay widths into $t \bar{t}$ quarks. The dependence on $\left|A_{\tau}\right|$ is also very weak, as long as $\left|A_{\tau}\right| \gtrsim 200 \mathrm{GeV}$. However, sending $\left|A_{\tau}\right| \rightarrow 0$ removes $\mathrm{CP}-$ violation from the $\tilde{\tau}$ sector and also greatly reduces the couplings of Higgs bosons to $\tilde{\tau}$ pairs, see Eq.(20); hence all $\mathrm{CP}$-odd asymmetries become very small in this limit. The dependence on $|\mu|$ (dot-dashed curve) is somewhat stronger than that on $\left|A_{\tau}\right|$, however, we again find a maximal asymmetry $\gtrsim 0.5$ unless $|\mu|$ is reduced by more than a factor of 3 from its standard input value of $2 \mathrm{TeV}$. Finally, the maximal asymmetry remains finite even as $\left|A_{t}\right|=\left|A_{b}\right| \rightarrow 0$, as shown by the dotted curve. In this limit there is no $\mathrm{CP}$-violation in the Higgs sector, but we already saw in Fig. 娄 that $\mathrm{CP}-$ violation in the $\tilde{\tau}$ sector by itself is sufficient to produce a large value of this asymmetry. 


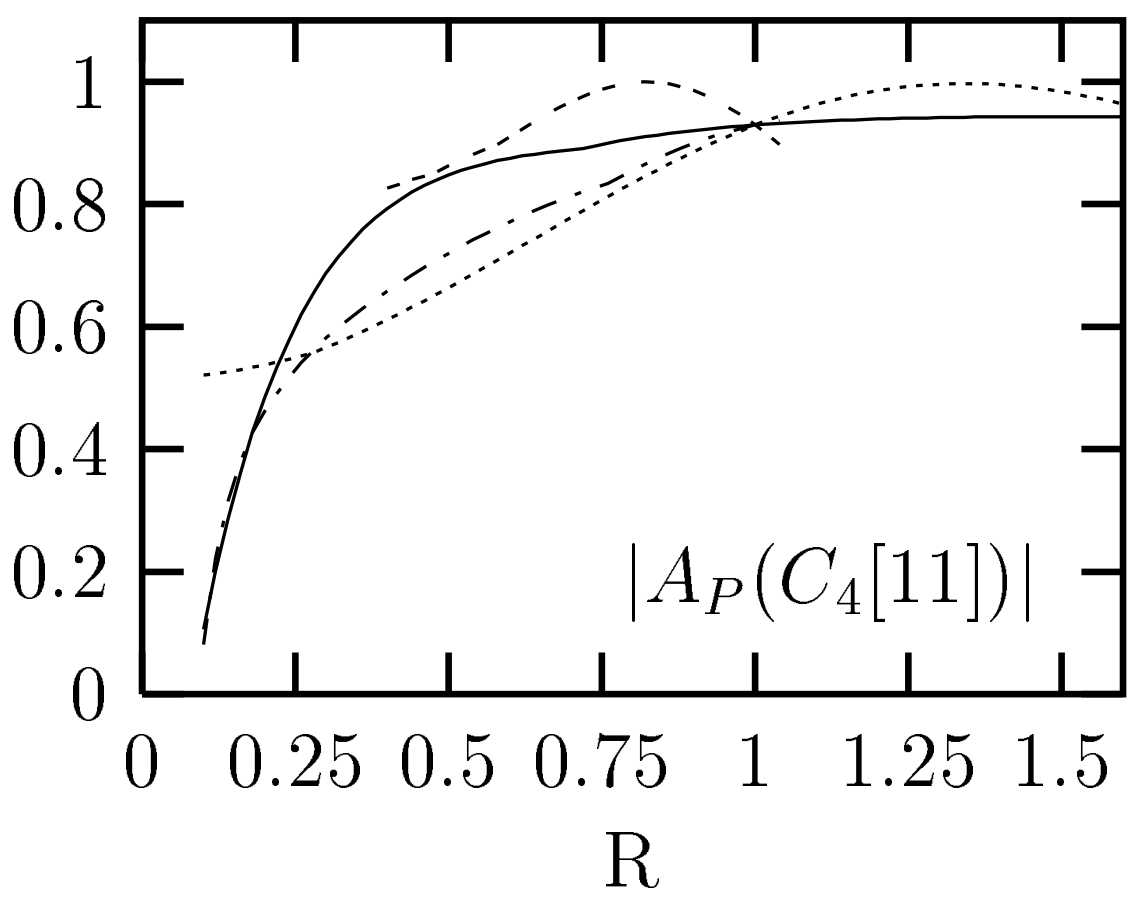

Figure 5: The maximal value of $\left|\mathcal{A}_{P}\left(C_{4}[11]\right)\right|$ is shown for $\Phi_{A \mu}=\pi / 2$ and $\Phi_{A_{\tau}}=4 \pi / 3$, where "maximal" means that the optimal value of $\sqrt{s}$ has been taken. The other input parameters have been fixed to their "standard" values, except that one of these parameters has been varied along each curve. The $x$-axis is the ratio of this parameter to its "standard" value: for the solid curve, $R=\left|A_{\tau}\right| /(0.5 \mathrm{TeV})$; for the dashed curve, $R=\tan \beta / 10$; for the dot-dashed curve, $R=|\mu| /(2 \mathrm{TeV})$; and for the dotted curve, $R=\left|A_{t}\right| /(1 \mathrm{TeV})$. The upper bounds on $\tan \beta$ and $|\mu|$ come from the requirement $m_{\tilde{\tau}_{1}} \geq 80 \mathrm{GeV}$, while the lower bound on $\tan \beta$ comes from the bound $m_{H_{1}} \geq 111$ GeV.

Unfortunately studies of $\tilde{\tau}_{1}$ pair production alone do not suffice to completely determine the parameters of the $\tilde{\tau}$ sector. Even under the most favorable circumstances such studies can only determine the values of four real parameters: the mass $m_{\tilde{\tau}_{1}}$, the mixing angle $\theta_{\tau}$ (these two parameters can already be determined from VV correlations), and the couplings of the two heavy Higgs bosons to $\tilde{\tau}_{1}$ pairs. On the other hand, the $\tilde{\tau}$ mass matrix depends on 6 real parameters $\left(m_{\tilde{\tau}_{L}}, m_{\tilde{\tau}_{R}},\left|A_{\tau}\right|,|\mu|, \tan \beta\right.$ and $\left.\Phi_{A_{\tau}}\right)$. Moreover, if the mixing between $\mathrm{CP}$-even and $\mathrm{CP}$-odd Higgs states is small, $C_{4}[11]$ and $C_{16}[11]$ essentially only depend on the product of the couplings of the two heavy Higgs bosons to $\tilde{\tau}_{1}$ pairs, since in such a situation the product of mixing angles $O_{1 k} O_{2 l}$ will be sizable only for $k \neq l$. In our numerical example this is trivially true for $\Phi_{A \mu}=0$, but is also approximately correct for $\Phi_{A \mu}=\pi / 2$, where $H_{2}$ is predominantly $\mathrm{CP}$-odd while $H_{3}$ is mostly $\mathrm{CP}$-even. Fortunately the total cross section for $\tilde{\tau}_{1}$ pair production 
for $\sqrt{s} \simeq m_{A}$ should depend on a different combination of Higgs couplings, so that it might still be possible to determine the couplings of both heavy Higgs states to $\tilde{\tau}_{1}$ pairs.

If mixed $\tilde{\tau}_{1}^{ \pm} \tilde{\tau}_{2}^{\mp}$ pairs are accessible, many more quantities become measurable. We saw above that studies of $\tilde{\tau}_{1}$ pair production should allow to determine 4 real parameters in the $\tilde{\tau}$ sector. In principle the total $\tilde{\tau}_{1}^{ \pm} \tilde{\tau}_{2}^{\mp}$ cross section, measured in the vicinity of the heavy Higgs poles, should then be sufficient to completely fix the parameters of the $\tilde{\tau}$ sector. However, this is only true if we assume that the masses and mixing angles of the neutral Higgs bosons are known. In addition, we have to assume that $\tilde{\tau}$ pair production is indeed described by the MSSM, and that there are no other diagrams contributing; for example, in the presence of slepton flavor mixing, $t$-channel diagrams might contribute [34]. Analyses of the new asymmetries that become available if $\tilde{\tau}_{1} \tilde{\tau}_{2}$ pairs can be produced can then be used to check the consistency of the framework followed in this paper. Moreover, (some of) these new asymmetries should also have quite different systematic uncertainties than the quantities discussed so far.

In particular, we now have the opportunity to study rate asymmetries in addition to polarization asymmetries. All the $\mathrm{SS}$ rate asymmetries, i.e. the two $\mathrm{CP}$-even asymmetries $\mathcal{A}_{R}\left(C_{4}[12]\right)$ and $\mathcal{A}_{R}\left(C_{16}[12]\right)$, and the two $\mathrm{CP}$-odd asymmetries $\mathcal{A}_{R}\left(C_{3}[12]\right)$ and $\mathcal{A}_{R}\left(C_{15}[12]\right)$, are in principle sensitive to $\mathrm{CP}$ violation in the tau slepton system even without reconstruction of the production plane. However, we already saw at the beginning of Sec. 4.2 that $\left[C_{3}[12]\right]$ is always very small in the MSSM with $m_{A}^{2} \gg m_{Z}^{2}$. Moreover, we find numerically that $A_{R}\left(C_{16}[12]\right)$ is relatively insensitive to $\Phi_{A_{\tau}}$, in particular if there is no $\mathrm{CP}$-violation in the Higgs sector. In Fig. 6 we therefore show $A_{R}\left(C_{4}[12]\right)$ (top row) and $A_{R}\left(C_{15}[12]\right)$ (bottom row).

The first of these asymmetries is even under both $\mathrm{CP}$ and $\mathrm{CP} \tilde{\mathrm{T}}$. It is therefore nonzero even if $\Phi_{A \mu}=\Phi_{A_{\tau}}=0$, and also remains finite far away from the heavy Higgs poles; recall that this asymmetry is normalized to the (unpolarized) squared Higgs boson exchange contribution only, see Eq. (49). However, this is only true for completely (transversely) polarized beams; and even in this case the number of events will become very small as one moves away from the Higgs poles. Nevertheless measuring this asymmetry in the vicinity of the pole should yield useful information. Note that in the absence of $\mathrm{CP}$-violation in the Higgs sector, the asymmetry depends only on $\cos \Phi_{A_{\tau}}$, i.e. it becomes invariant under $\Phi_{A_{\tau}} \rightarrow 2 \pi-\Phi_{A_{\tau}}$; this is true for all other $\mathrm{CP}$-conserving asymmetries as well. However, this degeneracy is broken if $\Phi_{A \mu} \neq 0$. Generally we find that CP-violation in the Higgs sector increases the sensitivity to the rephasing invariant phase in the $\tilde{\tau}$ sector.

This is true also for $A_{R}\left(C_{15}[12]\right)$. In fact, this asymmetry is very small if there is no $\mathrm{CP}-$ violation in the Higgs sector. The reason is that the products of couplings $O_{2 k} O_{2 l}$ and $O_{1 k} O_{1 l}$ appearing in this asymmetry, see Eq. (44), are almost proportional to $\delta_{k l}$ if the heavy $\mathrm{CP}$-even and $\mathrm{CP}$-odd Higgs states do not mix, while the combination $\mathcal{D}_{k l}$ of Higgs boson propagators vanishes for $k=l$. The interference between the heavy and light $\mathrm{CP}$-even Higgs states then at best gives an asymmetry of order $10^{-3}$, which is not measurable with realistic luminosities. On the other hand, once CP is violated in the Higgs sector, this asymmetry can become large. However, since it is CPT-odd, it decreases quickly as we move away from the Higgs poles. Moreover, the results of Fig. 6 indicate that this asymmetry is not very sensitive to $\Phi_{A_{\tau}}$ even if $\Phi_{A \mu} \neq 0$. 
(a) $\mathbf{A}_{\mathbf{R}}\left(\mathbf{C}_{4}[12]\right), \Phi_{\mathrm{A} \mu}=\mathbf{0}$

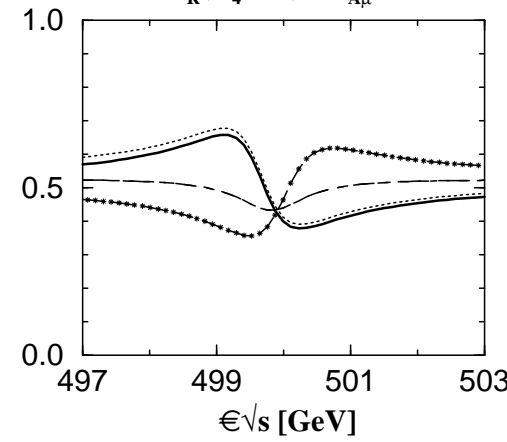

(c) $\mathbf{A}_{\mathrm{R}}\left(\mathbf{C}_{15}[12]\right), \Phi_{\mathrm{A} \mu}=0$

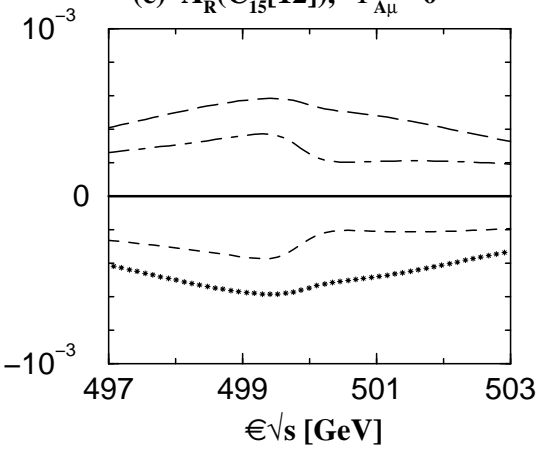

(b) $\mathbf{A}_{\mathbf{R}}\left(\mathbf{C}_{4}[12]\right), \Phi_{\mathrm{A} \mu}=\pi / 2$

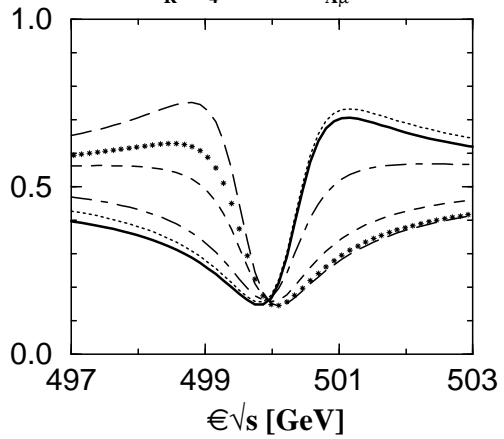

(d) $\mathbf{A}_{\mathbf{R}}\left(\mathbf{C}_{15}[\mathbf{1 2}]\right), \Phi_{\mathrm{A \mu}}=\pi / 2$

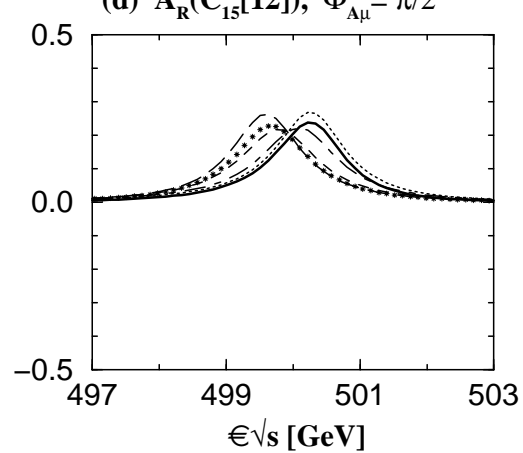

Figure 6: The $\sqrt{s}$ dependence of the CP-even $S S$ rate asymmetry $\mathcal{A}_{R}\left(C_{4}[12]\right)$ for $(a) \Phi_{A}=0$ and (b) $\Phi_{A}=\pi / 2$, and the $C P-$ odd $S S$ rate asymmetry $\mathcal{A}_{R}\left(C_{15}[12]\right)$ for (c) $\Phi_{A}=0$ and (d) $\Phi_{A}=\pi / 2$. Parameters and notations are as in Fig. 3.

\subsection{With direct reconstruction of the production plane}

If the production plane of the tau slepton pair can be reconstructed efficiently, the SS asymmetries related to $C_{13}$ and $C_{14}$ become accessible. More importantly, all SV correlations can now be studied. These asymmetries are linear in Higgs propagators, which implies that they are less sensitive to the strength of the Yukawa coupling of the muon than the SS correlations are. Moreover, in principle asymmetries $\propto \Re e\left[D_{H_{k}}\right]$ allow one to "switch off" the contribution from Higgs mass eigenstate $H_{k}$ simply by setting $\sqrt{s}=m_{H_{k}}$, since the real part of the Higgs propagator vanishes there. In this manner one might be able to cleanly isolate the contribution from the second heavy Higgs boson. In this section, based on the parameter sets (51) and (53), we investigate quantitatively whether the SV asymmetries can provide us with additional useful information on the $\tilde{\tau}$ sector.

In principle SV correlations can already be studied in polarization asymmetries in the $\tilde{\tau}_{1}^{+} \tilde{\tau}_{1}^{-}$ channel. Unfortunately we find numerically that these asymmetries are always rather small, with absolute value $\lesssim 0.2$. This is true also for polarization asymmetries in the mixed $\tilde{\tau}_{1}^{ \pm} \tilde{\tau}_{2}^{\mp}$ channel. We are thus left with the 8 rate asymmetries $\mathcal{A}_{R}\left(C_{n}[12]\right)(n=5-12)$. Eq. (45) shows that all these asymmetries depend on the parameters of the $\tilde{\tau}$ sector through the combination 
of couplings

$$
I_{k} \equiv \Im m\left(V_{k ; 12} Q_{12}^{Z *}\right) \text {, }
$$

as well as through the masses of the $\tilde{\tau}$ sleptons. A closer look at the explicit expressions for these rate asymmetries shows that the four asymmetries $\mathcal{A}_{R}\left(C_{n}[12]\right)(n=6,7,10,11)$ are proportional to the small numerical factor $4 s_{W}^{2}-1 \approx-0.07$ for $s_{W}^{2} \approx 0.233$; this comes from the vector coupling of the $Z$ boson to muons. We will therefore not consider these asymmetries, and instead concentrate on the remaining four asymmetries, which stem from the axial vector coupling of the $Z$ boson to muons. They satisfy the proportionality relations:

$$
\begin{aligned}
& \mathcal{A}_{R}\left(C_{5}[12]\right) \propto-O_{2 k} \Im \mathrm{m}\left[D_{H_{k}}\right] I_{k}, \\
& \mathcal{A}_{R}\left(C_{8}[12]\right) \propto+s_{\beta} O_{1 k} \Im \mathrm{m}\left[D_{H_{k}}\right] I_{k}, \\
& \mathcal{A}_{R}\left(C_{9}[12]\right) \propto+s_{\beta} O_{1 k} \Re \mathrm{e}\left[D_{H_{k}}\right] I_{k}, \\
& \mathcal{A}_{R}\left(C_{12}[12]\right) \propto-O_{2 k} \Re \mathrm{e}\left[D_{H_{k}}\right] I_{k} .
\end{aligned}
$$

(a) $\mathbf{A}_{\mathbf{R}}\left(\mathbf{C}_{5}[\mathbf{1 2}]\right), \Phi_{A \mu}=\mathbf{0}$

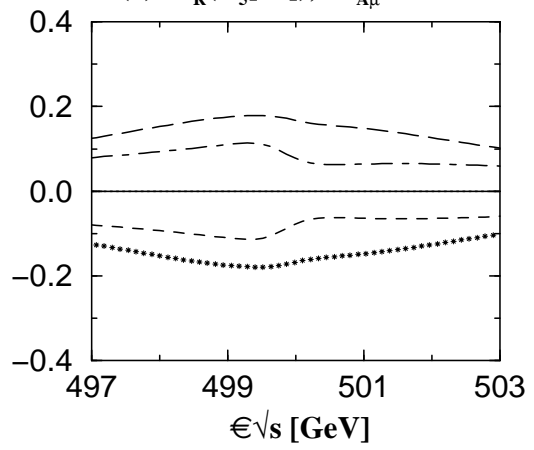

(c) $\mathbf{A}_{\mathrm{R}}\left(\mathbf{C}_{8}[\mathbf{1 2}]\right), \Phi_{\mathrm{A \mu}}=\mathbf{0}$

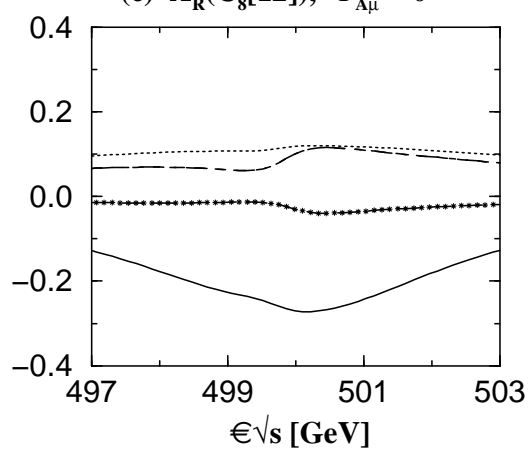

(b) $\mathbf{A}_{\mathrm{R}}\left(\mathbf{C}_{5}[\mathbf{1 2}]\right), \Phi_{\mathrm{A} \mu}=\pi / 2$

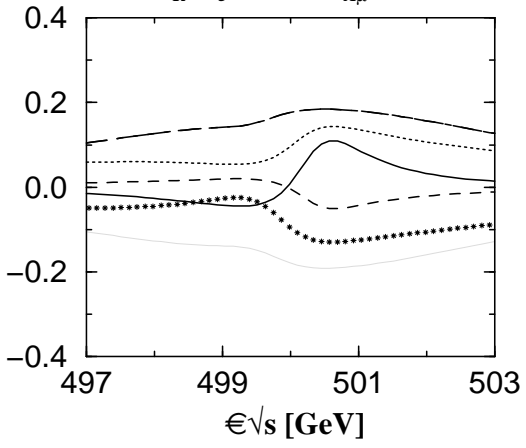

(d) $\mathbf{A}_{\mathbf{R}}\left(\mathbf{C}_{8}[\mathbf{1 2}]\right), \Phi_{\mathrm{A} \mu}=\pi / 2$

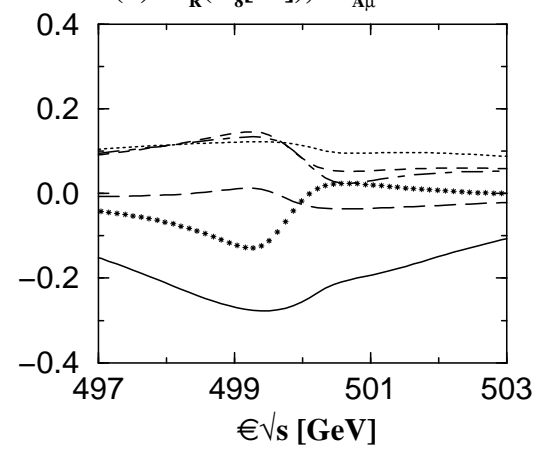

Figure 7: The $\sqrt{s}$ dependence of the $C P$-violating $S V$ rate asymmetry $\mathcal{A}_{R}\left(C_{5}[12]\right)$ for (a) $\Phi_{A \mu}=0$ and (b) $\Phi_{A \mu}=\pi / 2$, and the $C P$-conserving $S V$ rate asymmetry $\mathcal{A}_{R}\left(C_{8}[12]\right)$ for (c) $\Phi_{A \mu}=0$ and (d) $\Phi_{A \mu}=\pi / 2$, respectively. Notations and parameters are as in Fig. $\mathrm{G}$.

These relations show that the $\mathrm{CP}$-conserving asymmetries $\mathcal{A}_{R}\left(C_{8,9}\right)$ involve the $\mathrm{CP}$-odd components $\left(O_{1 k}\right)$ of the Higgs bosons, while the $\mathrm{CP}$-violating asymmetries $\mathcal{A}_{R}\left(C_{5,12}\right)$ involve the 
$\mathrm{CP}$-even components $\left(\mathrm{O}_{2 k}\right)$ of the Higgs bosons. This means that in the absence of $\mathrm{CP}$ violation in the Higgs sector, only one of the two heavy Higgs bosons contributes to a given asymmetry. In our case this remains approximately true even if $\Phi_{A \mu} \neq 0$, since, as noted earlier, the quantity $\sin \left(2 \alpha_{H}\right)$, which measures the strength of $\mathrm{CP}$-violation in the Higgs sector, is quite small. In other words, in our examples one does not even have to tune $\sqrt{s} \simeq m_{H_{k}}$ in order to isolate the contribution of a specific Higgs boson.

Note also that the two CP $\tilde{T}$-odd asymmetries $\mathcal{A}_{R}\left(C_{5,8}[12]\right)$ have their peaks at the heavy Higgs boson poles, but they are suppressed far away from those poles due to $\Im \mathrm{m}\left[D_{H_{k}}\right]$. However, Fig. (7) shows that these asymmetries only decrease relatively slowly as one moves away from the poles. This can be understood from the strong energy dependence of the total $\tilde{\tau}_{1} \tilde{\tau}_{2}$ production cross section shown in Fig. 3: the denominator of $\mathcal{A}_{R}\left(C_{5,8}[12]\right)$ also decreases quickly as one moves away from the poles.

(a) $\mathbf{A}_{\mathrm{R}}\left(\mathrm{C}_{9}[12]\right), \Phi_{\mathrm{A} \mu}=\mathbf{0}$

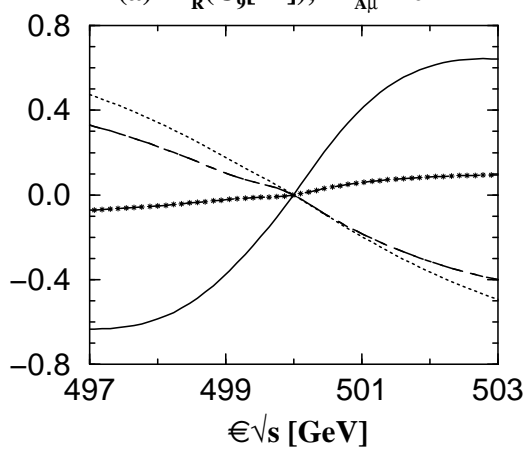

(c) $\mathbf{A}_{\mathrm{R}}\left(\mathbf{C}_{12}[\mathbf{1 2}]\right), \Phi_{\mathrm{A \mu}}=\mathbf{0}$

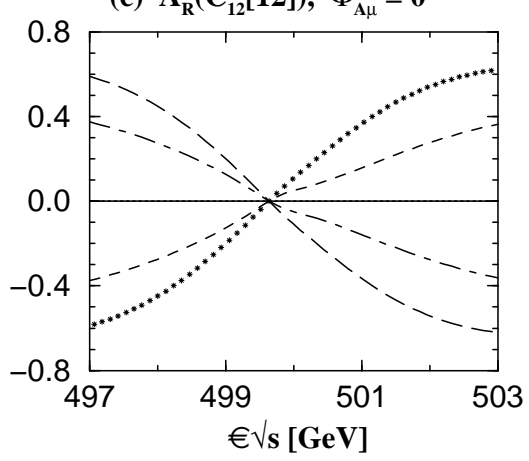

(b) $\mathbf{A}_{\mathrm{R}}\left(\mathbf{C}_{9}[12]\right), \Phi_{\mathrm{A} \mu}=\pi / 2$

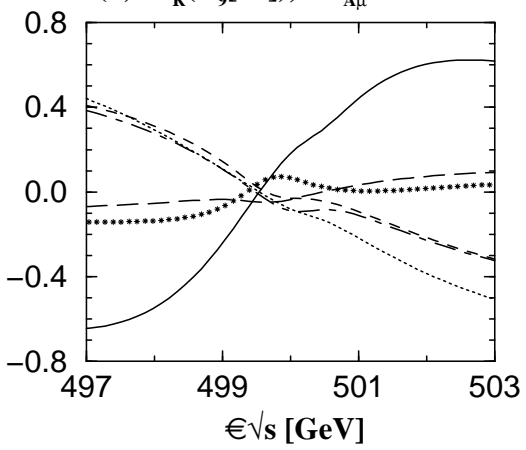

(d) $\mathbf{A}_{\mathrm{R}}\left(\mathbf{C}_{12}[12]\right), \Phi_{\mathrm{A} \mu}=\pi / 2$

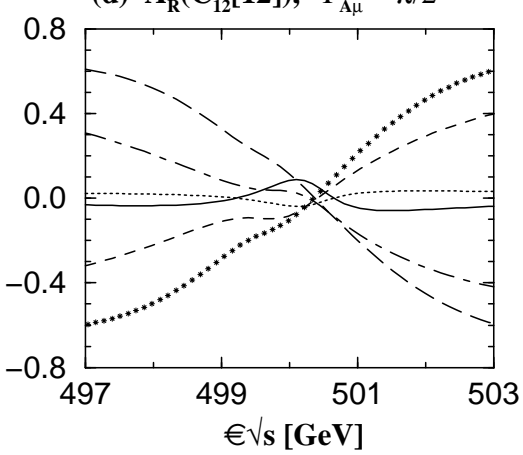

Figure 8: The $\sqrt{s}$ dependence of the $C P$-conserving $S V$ rate asymmetry $\mathcal{A}_{R}\left(C_{9}[12]\right)$ for (a) $\Phi_{A \mu}=0$ and (b) $\Phi_{A \mu}=\pi / 2$, and the $C P$-violating $S S$ rate asymmetry $\mathcal{A}_{R}\left(C_{12}[12]\right)$ for $(c)$ $\Phi_{A \mu}=0$ and (d) $\Phi_{A \mu}=\pi / 2$, respectively. Parameters and notation are as in Fig. 可.

On the contrary, Fig. 8 shows that the other two asymmetries $\mathcal{A}_{R}\left(C_{9,12}[12]\right)$, which are CPT-even, reach their extrema several GeV away from the Higgs poles. The numerators of 
these asymmetries actually reach their extrema at $\sqrt{s}=m_{H} \pm \Gamma_{H} / 2$, where $m_{H}$ and $\Gamma_{H}$ are a typical heavy Higgs mass and decay width, respectively. However, the rapid decrease of the denominator pushes the extrema further away from the Higgs poles. Note also that these asymmetries can attain larger values than the CP $\tilde{T}$-odd asymmetries shown in Fig. 7. However, while $\mathcal{A}_{R}\left(C_{5,8}[12]\right)$ can be measured if only one initial beam is (transversely) polarized, $\mathcal{A}_{R}\left(C_{9,12}[12]\right)$ can only be determined if both muon beams are polarized, one having a transverse and the other a longitudinal polarization. Together with the requirement that the event plane has to be reconstructed this means that measuring the asymmetries shown in Fig. 8 will probably pose the biggest challenge to both collider and detector.

\section{$6 \quad$ Summary and Conclusions}

We have performed a detailed, systematic investigation of the signals for $\mathrm{CP}$ violation in the neutral Higgs boson and tau-slepton systems through the production of tau slepton pairs in polarized $\mu^{+} \mu^{-}$collisions, $\mu^{-} \mu^{+} \rightarrow \tilde{\tau}_{i}^{-} \tilde{\tau}_{j}^{+}$with the labels $i, j=1,2$ for the two $\tau$ slepton mass eigenstates. We worked in the framework of the MSSM with exact R-parity and negligible flavor mixing. The relevant sources of $\mathrm{CP}$ violation can then be found in soft breaking terms associated with third generation sfermions, as well as the $\mu$-parameter. $\mathrm{CP}$ violation in the $\tilde{\tau}$ sector contributes to $\mathrm{CP}$-odd asymmetries at the tree-level. $\mathrm{CP}$ violation in the $\tilde{t}$ and/or $\tilde{b}$ sector leads to mixing between $\mathrm{CP}$-even and $\mathrm{CP}$-odd Higgs current eigenstates. Even though this $\mathrm{CP}$-violating Higgs mixing only proceeds through loop diagrams, it can give rise to $\mathcal{O}(1)$ $\mathrm{CP}$-violating asymmetries even in the absence of other $\mathrm{CP}$ phases.

The expression for the cross section for $\tilde{\tau}$ pair production from a $\mu^{+} \mu^{-}$initial state with arbitrary (possibly transverse) polarization contains 16 terms. We have classified the behavior of these terms under $\mathrm{CP}$ and $\mathrm{CP} \tilde{\mathrm{T}}$ transformations. Terms with $\mathrm{CP}$-even polarization factors can contribute to $\mathrm{CP}$-violating rate asymmetries, i.e. differences between the cross sections for $\tilde{\tau}_{1}^{+} \tilde{\tau}_{2}^{-}$and $\tilde{\tau}_{1}^{-} \tilde{\tau}_{2}^{+}$production. On the other hand, terms with $\mathrm{CP}$-odd polarization factors contribute to $\mathrm{CP}$-violating polarization asymmetries that can be probed already in $\tilde{\tau}_{1}$ pair production. In some cases it can also be interesting to construct asymmetries from these terms which are even under a CP transformation. These asymmetries are nonzero even in the absence of $\mathrm{CP}$ violation, but they can nevertheless help to determine the to date unknown parameters in the problem, including phases.

It is reasonable to assume that all the properties of the neutral Higgs bosons are determined beforehand, e.g. through the study of $\mu^{+} \mu^{-} \rightarrow f \bar{f}$ production near the Higgs poles, where $f=\tau, b$ or $t$. The remaining task is thus the determination of the parameters in the $\tilde{\tau}$ sector. As a first step one might want to determine the masses of the accessible $\tilde{\tau}$ states, as well as the $\tilde{\tau}_{L}-\tilde{\tau}_{R}$ mixing angle $\theta_{\tau}$ through the study of $\tilde{\tau}$ pair production away from the Higgs poles. Here the cross section will be dominated by gauge interactions, and is thus completely determined by these parameters. The mixing angle $\theta_{\tau}$ also affects the polarization of the $\tau$ leptons produced in $\tilde{\tau}$ decays [35. Of course, these measurements can also be performed at an $e^{+} e^{-}$collider. 
(a) $A_{P}\left(C_{4}\{11\}\right), \Phi_{A \mu}=0$

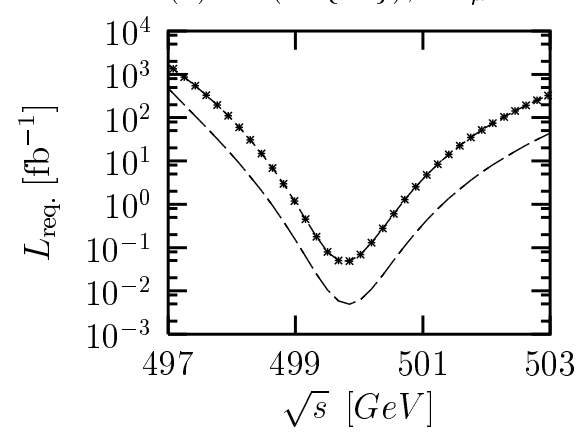

(c) $A_{R}\left(C_{5}[12]\right), \Phi_{A \mu}=0$

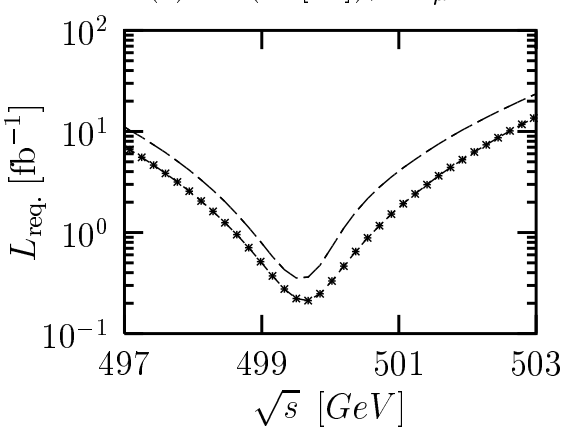

(b) $A_{P}\left(C_{4}\{11\}\right), \Phi_{A \mu}=\frac{\pi}{2}$

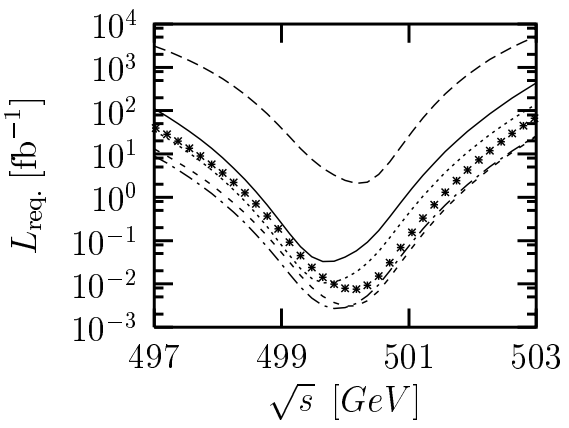

(d) $A_{R}\left(C_{5}[12]\right), \Phi_{A \mu}=\frac{\pi}{2}$

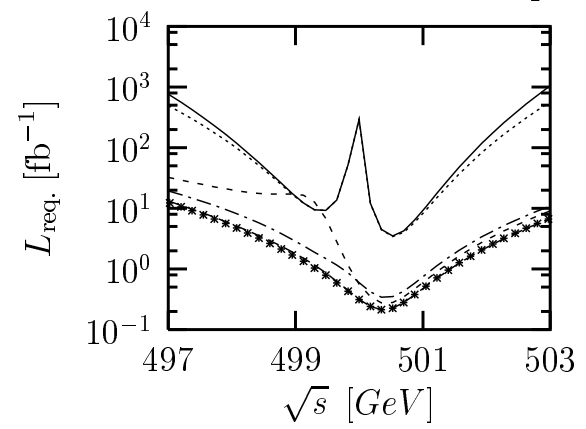

Figure 9: The $\sqrt{s}$ dependence of the integrated luminosity required to detect the asymmetries $\mathcal{A}_{P}\left(C_{4}[11]\right)(a, b)$ and $\mathcal{A}_{R}\left(C_{5}[12]\right)(c, d)$ with statistical significance of one standard deviation, for ideal beam polarization. The luminosity increases quadratically with the required number of standard deviations, and with the inverse of the degree of beam polarization. Parameters and notation are as in Fig. 3 .

$\tilde{\tau}$ pair production through gauge interactions can thus at best give three relations among the six real parameters appearing in the $\tilde{\tau}$ mass matrix. The remaining parameters can be determined if there are sizable Higgs exchange contributions. In particular, the longitudinal polarization asymmetry $\mathcal{A}_{P}\left(C_{4}[11]\right)$ is usually ideally suited for directly probing $\mathrm{CP}$-violation in the $\tilde{\tau}$ sector. One does not need to reconstruct the event plane to measure this asymmetry, nor are transversely polarized beams required. Moreover, this asymmetry is linear in the achievable polarization; this is of some importance, since the actual beam polarization might be significantly less than the ideal value of $100 \%$ assumed in the numerical results of Sec. 5 . Of course, we have to assume that $\tilde{\tau}$ pair production is kinematically allowed in the vicinity of the Higgs poles. Moreover, the ratio of vevs $\tan \beta$ must not be too small, since the couplings of both muons and tau (s)leptons to Higgs bosons scale essentially $\propto \tan \beta$. However, scenarios with very small $\tan \beta$ are essentially excluded by LEP Higgs searches anyway. The upper two frames in Fig. 9 show that, if these conditions are met and the $\mathrm{CP}$-violating phases are not very small, this asymmetry should indeed be measurable with the foreseen luminosity of about $10 \mathrm{fb}^{-1}$ per year. Here we show the integrated luminosity required to see a $1 \sigma$ deviation of this asymmetry from zero, for ideal beam polarization. The required luminosity scales quadratically with the required number of standard deviations as well as with the available polarization. For 
example, a value of $0.01 \mathrm{fb}^{-1}$ means that with a data set of $10 \mathrm{fb}^{-1}$ and $35 \%$ beam polarization one can see an $11 \sigma$ effect, i.e. this asymmetry would be measurable with relative precision of about 9\%. We saw in Fig. 5 that this conclusion should remain true over a wide region of parameter space.

Note, however, that this asymmetry might be "accidentally" suppressed, even though there are large $\mathrm{CP}$-violating phases in both the $\tilde{\tau}$ and $\tilde{t}$ sectors. It is therefore important to try and measure several independent $\mathrm{CP}$-violating asymmetries. Moreover, analyses of $\tilde{\tau}_{1}$ pair production in the MSSM can at best determine the couplings of $\tilde{\tau}_{1}$ pairs to the two heavy neutral Higgs bosons. LEP search limits on $m_{\tilde{\tau}_{1}}$ together with the bound $m_{H_{1}} \lesssim 130 \mathrm{GeV}$ imply that the exchange of the lightest neutral Higgs boson $H_{1}$ contributes negligibly to $\tilde{\tau}$ pair production. Simple parameter counting then implies that, in the absence of any prior knowledge of the parameters of the mass matrix, one will have to study $\tilde{\tau}_{1} \tilde{\tau}_{2}$ production in order to completely determine the parameters of the $\tilde{\tau}$ mass matrix. Note that some of these parameters $(\tan \beta$ as well as $\mu$ ) also appear in other terms in the MSSM Lagrangian. It is thus possible that their values will be known (with some error) beforehand. Even in that case it would be important to determine them independently from $\tilde{\tau}$ pair production, in order to confirm that the MSSM with the given assumptions can indeed describe this process. Of course, this will only be possible if $m_{A}>m_{\tilde{\tau}_{1}}+m_{\tilde{\tau}_{2}}$.

Unfortunately we found that $\mathrm{CP}$-violating asymmetries that can be measured in the mixed $\tilde{\tau}_{1} \tilde{\tau}_{2}$ channel are smaller than $\mathcal{A}_{P}\left(C_{4}[11]\right)$, at least in the vicinity of the Higgs poles where the event rate is sizable. This is demonstrated by the lower two frames of Fig. 9, which shows the luminosity required to see a $1 \sigma$ deviation in the rate asymmetry $\mathcal{A}_{R}\left(C_{5}[12]\right)$. In most cases the minimal required luminosity is at least an order of magnitude larger than for $\mathcal{A}_{P}\left(C_{4}[11]\right)$. We saw in Sec. 5 that CP $\tilde{T}$-even asymmetries can be $\mathcal{O}(1)$ even in the mixed $\tilde{\tau}_{1} \tilde{\tau}_{2}$ channel. However, these asymmetries are proportional to the real parts of heavy Higgs boson propagators, which vanish near the poles. As a result, the minimal luminosity required for measuring these asymmetries (not shown) is similar to that required to measure $\mathcal{A}_{R}\left(C_{5}[12]\right)$, but would have to be taken at $\sqrt{s} \simeq m_{H} \pm \Gamma_{H}$, where $m_{H}$ and $\Gamma_{H}$ are the typical mass and total decay width of the heavy neutral Higgs bosons. It thus seems unlikely that studies of mixed $\tilde{\tau}_{1} \tilde{\tau}_{2}$ production can contribute significantly to the determination of the $\mathrm{CP}$-violating phase in the $\tilde{\tau}$ mass matrix. Fortunately the determination of all parameters of this mass matrix can be completed by measuring a single quantity in the $\tilde{\tau}_{1} \tilde{\tau}_{2}$ channel, for example the total cross section near the Higgs poles. We saw in Sec. 5 that this cross section is indeed often sizable, and should thus be readily measurable. A possible problem here might be the distinction between $\tilde{\tau}_{1}$ pair and $\tilde{\tau}_{1} \tilde{\tau}_{2}$ production. This should be fairly easy in the scenario we studied in Sec. 5, due to the large $\tilde{\tau}_{2}-\tilde{\tau}_{1}$ mass splitting, but could be more problematic if this mass splitting is small.

We thus see that in the chosen framework transverse beam polarization is not necessary to determine all the free parameters of the $\tilde{\tau}$ system. However, in order to test the model one has to over-constrain it, i.e. there should be more measurements than parameters. Also, generalizations of the model, e.g. allowing $\mathrm{R}$-parity violation and/or slepton flavor mixing, are conceivable. In that case the squared matrix element can still be written as in Eq. (33), but 
there will be additional contributions [36] to the expressions (26), and hence to the coefficients $C_{n}$. It is therefore important to measure as many different asymmetries and distributions as possible, including those that can only be accessed with transversely polarized beams. Only then will it be possible to fully exploit the physics potential of muon colliders to probe the details of supersymmetric models through scalar $\tau$ production.

\section{Acknowledgements}

The work of S.Y.C. was supported by a grant from the Korea Research Foundation (KRF-2000015-DS0009). The work of M.D. and B.G. was supported in part by the Deutsche Forschungsgemeinschaft. The work of J.S.L. was supported by the Japan Society for the Promotion of Science (JSPS).

\section{References}

[1] J.H. Christenson, J.W. Cronin, V.L. Fitch and R. Turlay, Phys. Rev. Lett. 13, 138 (1964); for reviews, see, for example, P.K. Kabir, The CP Puzzle, (Academic Press, London and New York, 1968); W. Grimus, Fortschr. Phys. 36, 201 (1988); E.A. Paschos and U. Türke, Phys. Rep. 178, 147 (1989); B. Winstein and L. Wolfenstein, Rev. Mod. Phys. 65, 1113 (1993); G.D. Barr et al., NA31 Collaboration, Phys. Lett. B317, 233 (1993); A. AlaviHarati et al., KTeV Collaboration, Phys. Rev. Lett. 83, 22 (1999); V. Fanti et al., NA48 Collaboration, Phys. Lett. B465, 335 (1999).

[2] For pedagogical introduction to $\mathrm{CP}$ violation in the $B$-meson system, see M. Neubert, Int. J. Mod. Phys. A11, 4173 (1996); A.J. Buras, hep-ph/9806471 and references therein. The to date most accurate measurements of $\mathrm{CP}$-violating asymmetries in $B$ decays are: BELLE Collab., A. Abashian et al., Phys. Rev. Lett. 86, 2509 (2001), hep-ex/0102018; BABAR Collab., B. Aubert et al., Phys. Rev. Lett. 86, 2515 (2001), hep-ex/0102030.

[3] A.D. Sakharov, Zh. Eksp. Teor. Fiz. Pis'ma 5, 32 (1967); JETP Lett. 91B, 24 (1967).

[4] M. Kobayashi and T. Maskawa, Prog. Theor. Phys. 49, 652 (1973).

[5] E. Witten, Nucl. Phys. B188, 513 (1981).

[6] C. Giunti, C.W. Kim and U.W. Lee, Mod. Phys. Lett. A6, 1745 (1991); U. Amaldi, W. de Boer and H. Fürstenau, Phys. Lett. B260, 447 (1991); P. Langacker and M. Luo, Phys. Rev. D 44, 817 (1991); J. Ellis, S. Kelley and D.V. Nanopoulos, Phys. Lett. B260, 131 (1991).

[7] S. Dimopoulos and D. Sutter, Nucl. Phys. B 452 (1995) 496; H. Haber, Proceedings of the 5th International Conference on Supersymmetries in Physics (SUSY'97), May 1997, ed. M. Cvetić and P. Langacker, hep-ph/9709450. 
[8] T. Ibrahim and P. Nath, Phys. Lett. B418, 98 (1998); Phys. Rev. D 57, 478 (1998); D 58, 019901 (1998) (E); ibid, 111301 (1998); W. Hollik, J.I. Illana, S. Rigolin, C. Schappacher, and D. Stockinger, Nucl. Phys. B439, 3 (1999); M. Brhlik, G.J. Good and G.L. Kane, ibid. D 59, 115004-1 (1999); S. Pokorski, J. Rosiek and C.A. Savoy, Nucl. Phys. B570, 81 (2000), hep-ph/9906206; A. Bartl, T. Gajdosik, W. Porod, P. Stochinger and H. Stremnitzer, Phys. Rev. D 60, 073003 (1999), hep-ph/9903402; E. Accomando, R. Arnowitt, and B. Dutta, ibid. D 61, 115003 (2000), hep-ph/9907446; T. Ibrahim and P. Nath, ibid. D 61, 095008 (2000), hep-ph/9907555.

[9] T. Falk and K.A. Olive, Phys. Lett. B 439, 71 (1998), hep-ph/9806236.

[10] M.P. Worah, Phys. Rev. Lett. 79, 3810 (1997), hep-ph/9704389; N. Rius and V. Sanz, Nucl. Phys. B570, 1555 (2000), hep-ph/9907460.

[11] D. Chang, W.-Y. Keung and A. Pilaftsis, Phys. Rev. Lett. 82, 900 (1999), erratum: 83, 3972 (1999), hep-ph/9811202 ; A. Pilaftsis, Phys. Lett. B471, 174 (1999), hepph/9909485; D. Chang, W.-F. Chang, and W.-Y. Keung, Phys. Lett. B478, 239 (2000), hep-ph/9910465.

[12] A. Pilaftsis, Phys. Lett. B435, 88 (1998), hep-ph/9805373; Phys. Rev. D 58, 096010 (1998), hep-ph/9803297.

[13] A. Pilaftsis and C.E.M. Wagner, Nucl. Phys. B553, 3 (1999), hep-ph/9902371.

[14] D.A. Demir, Phys. Rev. D 60, 055006 (1999), hep-ph/9901389.

[15] S.Y. Choi, M. Drees and J.S. Lee, Phys. Lett. B481, 57 (2000), hep-ph/0002287.

[16] M. Carena, J. Ellis, A. Pilaftsis and C.E.M. Wagner, Nucl. Phys. B586, 92 (2000), hep $\mathrm{ph} / 0003180$.

[17] S.Y. Choi and M. Drees, Phys. Rev. Lett. 81, 5509 (1998).

[18] A. Pilaftsis, Phys. Rev. Lett. 77, 4996 (1997), hep-ph/9603328; K.S. Babu, C. Kolda, J. March-Russell and F. Wilczek, Phys. Rev. D 59, 016004 (1999), hep-ph/9804355; J.F. Gunion and J. Pliszka, Phys. Lett. B444, 136 (1998), hep-ph/9809306; C.A. Boe, O.M. Ogreid, P. Osland and J. Zhang, Eur. Phys. J. C9, 413 (1999), hep-ph/9811505; B. Grzadkowski, J.F. Gunion and J. Kalinowski, Phys. Rev. D 60, 075011 (1999), hep $\mathrm{ph} / 9902308$.

[19] S.Y. Choi and J.S. Lee, Phys. Rev. D 61, 015003 (2000), hep-ph/9907496.

[20] S.Y. Choi and J.S. Lee, Phys. Rev. D 61, 111702 (2000), hep-ph/9909315; Phys. Rev. D 61, 115002 (2000), hep-ph/9910557; Phys. Rev. D 62, 036005 (2000), hep-ph/9912330; A. Dedes and S. Moretti, Nucl. Phys. B576, 29 (2000), hep-ph/9909418; E. Asakawa, S.Y. Choi, K. Hagiwara and J.S. Lee, Phys. Rev. D 62, 115005 (2000). 
[21] E. Asakawa, S.Y. Choi and J.S. Lee, Phys. Rev. D 63, 015012 (2001).

[22] V. Barger, M.S. Berger, J.F. Gunion and T. Han, Phys. Rep. 281, 1 (1997).

[23] V. Barger, M.S. Berger, J.F. Gunion, and T. Han, Phys. Rev. Lett. 75, 1462 (1995); J.F. Gunion, in Proceedings of the 5th International Conference on Physics Beyond the Standard Model, Balholm, Norway, 1997, edited by G. Eigen, P. Osland and B. Stugu (AIP, Woodbury, New York, 1997), p. 234.

[24] D. Atwood and A. Soni, Phys. Rev. D 52, 6271 (1995), hep-ph/9505233; A. Pilaftsis, Phys. Rev. Lett. 77, 4996 (1996), hep-ph/9603328; Nucl. Phys. B504, 61 (1997), hepph/9702393.

[25] B. Grzadkowski, J.F. Gunion and J. Pliszka, Nucl. Phys. B583, 49 (2000), hep ph/0003091; hep-ph/0004034 and references therein.

[26] A. Bartl, H. Eberl, S. Kraml, W. Majerotto and W. Porod, Phys. Rev. D 58, 115002 (1998), hep-ph/9805248.

[27] M. Nowakowski and A. Pilaftsis, Phys. Lett. B245, 185 (1990).

[28] N. Arkani-Hamed, J.L. Feng and L.J. Hall, Nucl. Phys. B505, 3 (1997); D. Bowser-Chao and W.-Y. Keung, Phys. Rev. D 56, 3924 (1997).

[29] S.Y. Choi and M. Drees, Phys. Lett. B435, 356 (1998), hep-ph/9805474.

[30] F. Gabbiani, E. Gabrielli, A. Masiero and L. Silvestrini, Nucl. Phys. B477, 321 (1996), hep-ph/9604387.

[31] S. Coleman and E. Weinberg, Phys. Rev. D 7, 1888 (1973); Y. Okada, M. Yamaguchi and T. Yanagida, Prog. Theor. Phys. 85, 1 (1991); Phys. Lett. B262, 54 (1991); J. Ellis, G. Ridolfi and F. Zwirner, Phys. Lett. B257, 83 (1991); B262, 477 (1991).

[32] K. Hagiwara and D. Zeppenfeld, Nucl. Phys. B274, 1 (1986).

[33] J.L. Feng and M.J. Strassler, Phys. Rev. D 55, 1326 (1997), ‘hep-ph/9606477; S.Y. Choi, A. Djouadi, H.S. Song and P.M. Zerwas, Eur. Phys. J. C8, 669 (1999), hep-ph/9812236.

[34] J. Hisano, M.M. Nojiri, Y. Shimizu and M. Tanaka, Phys. Rev. D 60, 055008 (1999), hep-ph/9808410.

[35] M.M. Nojiri, Phys. Rev. D 51, 6281 (1995), hep-ph/9412374.

[36] CP-violating asymmetries in the context of a model with broken R-parity are discussed by M. Chemtob and G. Moreau, Phys. Lett. B448, 57 (1999), hep-ph/9808428. 